\title{
Study of a Complete Abstract Differential Equation of Elliptic Type with Variable Operator Coefficients, I
}

\author{
Angelo FAVINI, Rabah LABBAS, \\ Keddour LEMRABET, and Boubaker-Khaled SADALLAH \\ Università degli Studi di Bologna \\ Dipartimento di Matematica \\ Piazza di Porta S. \\ Donato 5, 40126 Bologna - Italy \\ favini@dm.unibo.it \\ Laboraoire AMNEDP \\ Faculté des Maths, USTHB \\ BP 32, El Alia, Bab Ezzouar \\ 16111 Alger - Algeria \\ keddourlemrabet@yahoo.fr \\ Laboratoire de Mathématiques Appliquées \\ Université du Havre \\ U.F.R Sciences et Techniques, B.P 540 \\ 76058 Le Havre - France \\ rabah.labbas@univ-lehavre.fr \\ Laboratoire EDP et Hist. Maths \\ Ecole Normale Supérieure \\ 16050 Kouba, Alger - Algeria \\ sadallah@ens-kouba.dz
}

\begin{abstract}
The aim of this first work is the resolution of an abstract complete second order differential equation of elliptic type with variable operator coefficients set in a small length interval. We obtain existence, uniqueness and maximal regularity results under some appropriate differentiability assumptions combining those of Yagi [13] and Da Prato-Grisvard [6]. An example for the Laplacian in a regular domain of $\mathbb{R}^{3}$ will illustrate the theory. A forthcoming work (Part II) will complete the present one by the study of the Steklov-Poincaré operator related to this equation when the length $\delta$ of the interval tends to zero.
\end{abstract}

Key words: abstract differential equations of second order, variable operator coefficients, mixed boundary conditions, maximal regularity, compatibility conditions.

2000 Mathematics Subject Classification: 34G10, 34K10,34K30, 35J25, 44A45, 47D03. 


\section{Introduction}

\subsection{The abstract equation and hypotheses}

Recently, many works have dealt with the resolution of the complete abstract differential equation of second order

$$
\left\{\begin{array}{l}
\left.u^{\prime \prime}(t)+p u^{\prime}(t)+q u(t)=f(t), \quad t \in\right] 0, \delta[ \\
u(0)=\varphi_{0} \\
u(\delta)=\varphi_{\delta}
\end{array}\right.
$$

with variable operator coefficients in a Banach space $E$. Some optimal results about the existence, uniqueness and maximal regularity have been established in the framework of the following spaces:

$$
\left\{\begin{array}{l}
\left.C^{2 \theta}([0, \delta] ; E), \quad 2 \theta \in\right] 0,1[, \quad E \text { is an arbitrary Banach space, } \\
\left.L^{p}(0, \delta ; E), \quad p \in\right] 1, \infty[, \quad E \text { is an UMD Banach space, }
\end{array}\right.
$$

when the closed linear operators $(p, D(p))$ and $(q, D(q))$ satisfy some ellipticity and commutativity hypotheses (see, for example, $[3,4]$ ).

In this paper, we consider the complete abstract differential equation of second order

$$
\left\{\begin{array}{l}
\left.u^{\prime \prime}(t)+p(t) u^{\prime}(t)+q(t) u(t)-\lambda u(t)=f(t), \quad t \in\right] 0, \delta[ \\
u(0)=\varphi \\
u^{\prime}(\delta)=\psi
\end{array}\right.
$$

where

- $\lambda$ is a positive real number,

- $\left.f \in C^{2 \theta}([0, \delta], E), 2 \theta \in\right] 0,1[$,

- $(q(t))_{t \in[0, \delta]}$ is a family of closed linear operators of domains $D(q(t))$ (which may be not dense),

- for each $t \in[0, \delta], p(t)$ is a bounded linear operator.

- $\delta$ is a small real positive number. (In the second part of this paper, we will focus on the limit problem as $\delta$ tends to zero.)

The main difference between (1) and (2) is that the operators coefficients $q(t)$ are variable, whereas $p(t)$ is a perturbation.

Our purpose is to extend the results obtained for Problem (1) to Problem (2) by building explicitly a representation of the solution $u(t)$ and studying its optimal regularity. Some natural compatibility conditions related to the equation will be taken into account. 
The techniques used here are essentially based on the Dunford functional calculus and the methods applied in $[1,6,9,13]$.

Recall, briefly, the case when $p(t) \equiv 0, \lambda=0$, and $q(t)$ is a constant operator satisfying the natural ellipticity hypothesis

$$
\mathbb{R}^{+} \subset \rho(q) \text { and } \exists C>0, \forall z \geq 0\left\|(q-z I)^{-1}\right\| \leq \frac{C}{1+z},
$$

which is verified in a sector of the form

$$
\Pi_{\theta_{0}, r_{0}}=\left\{z \in \mathbb{C}^{*}:|\arg (z)| \leq \theta_{0}\right\} \cup\left\{z \in \mathbb{C}:|z| \leq r_{0}\right\}
$$

with some small $\theta_{0}>0$ and $r_{0}>0$. (Here, $\rho(q)$ is the resolvent set of $q$. )

The representation of the solution $u$ is given by the formula

$$
\begin{aligned}
u(t)= & \frac{1}{2 i \pi} \int_{\gamma} \frac{\cosh \sqrt{-z}(\delta-t)}{\cosh \sqrt{-z} \delta}(q-z I)^{-1} \varphi d z \\
& +\frac{1}{2 i \pi} \int_{\gamma} \frac{\sinh \sqrt{-z} t}{\sqrt{-z} \cosh \sqrt{-z} \delta}(q-z I)^{-1} \psi d z \\
& -\frac{1}{2 i \pi} \int_{\gamma} \int_{0}^{\delta} K_{\sqrt{-z}}(t, s)(q-z I)^{-1} f(s) d s d z
\end{aligned}
$$

with

$$
K_{\rho}(t, s)= \begin{cases}\frac{\sinh \rho s \cosh \rho(\delta-t)}{\rho \cosh \rho \delta} & \text { if } \quad 0 \leq s<t, \\ \frac{\sinh \rho t \cosh \rho(\delta-s)}{\rho \cosh \rho \delta} & \text { if } \quad t \leq s \leq \delta .\end{cases}
$$

The curve $\gamma$ is the retrograde oriented boundary of the sector $\Pi_{\theta_{0}, r_{0}}$.

We will deal with the case when the operators

$$
Q(t)=q(t)-\lambda I, \quad \lambda>0,
$$

are variable. The model we will present at the end of this paper considers realizations of $q(t)$ having $C^{2}$-coefficients and satisfying the Lopatinski-Shapiro conditions. On the other hand, we will consider some appropriate differentiability hypotheses on the resolvents of $q(t)$, combining those in Yagi [13] and Da Prato and Grisvard [6].

As mentioned previously, we assume that the operators $p(t)$ fulfill the estimate

$$
\exists C>0: \forall t \in[0, \delta] \quad\|p(t)\|_{L(E)} \leq C .
$$

Throughout this paper, the family of linear closed operators $(q(t))_{t \in[0, \delta]}$ of domains $D(q(t))$, not necessarily dense in $E$, enjoys the following three properties: 
(i)

$$
\exists M>0, \quad \forall z \geq 0, \forall t \in[0, \delta] \quad\left\|(q(t)-z I)^{-1}\right\|_{L(E)} \leq \frac{M}{1+z} .
$$

It is known that this assumption allows us to define the fractional powers of operators $(-Q(t))$.

(ii) For all $z \geq 0$, the application $t \mapsto(q(t)-z I)^{-1}$ defined on $[0, \delta]$ is in $C^{2}([0, \delta], L(E))$ and there exist $C>0$ and $\left.\left.\alpha \in\right] 1 / 4,1 / 2\right]$ such that

$$
\forall z \geq 0, \forall t \in[0, \delta] \quad\left\|q(t)(q(t)-z I)^{-1} \frac{d}{d t} q(t)^{-1}\right\|_{L(E)} \leq \frac{C}{z^{\alpha+1 / 2}} .
$$

Moreover there exist two constants $\eta$ and $\rho$ such that $\forall z \geq 0, \forall t, s \in[0, \delta]$

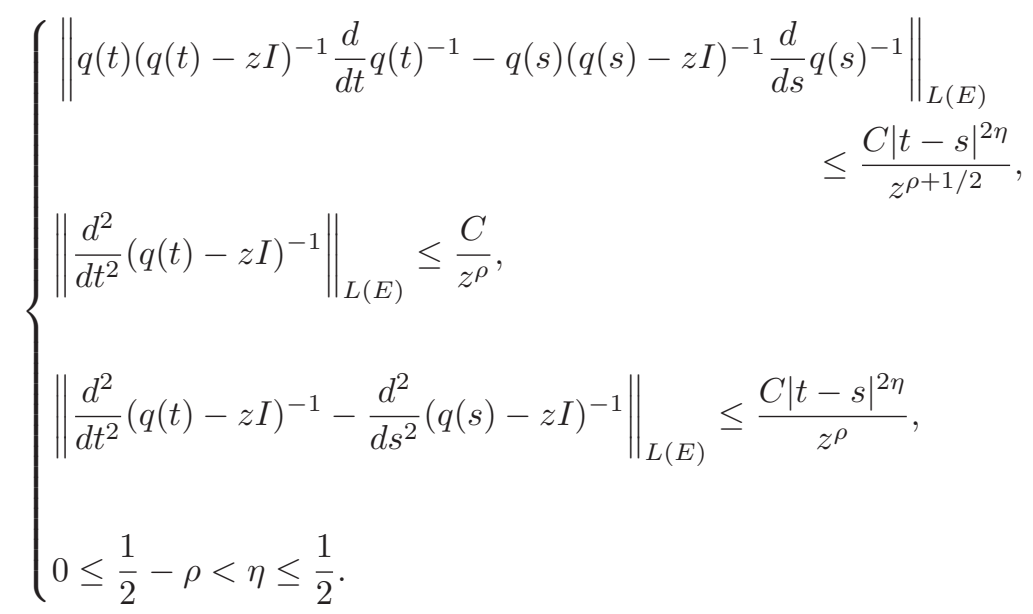

Remark 0.1. Observe that all the constants given above are independent of $t$ and $\delta$. On the other hand, it is clear, by using a classical argument of analytic continuation on the resolvent, that the previous assumptions hold true in the sector $\Pi_{\theta_{0}, r_{0}}$, and then, on $\gamma$. Furthermore, we can replace $z$ by $z+\lambda$.

\subsection{Comments and remarks on the hypotheses}

By the hypotheses (8) and (9) it follows that

$$
\left\|\frac{\partial}{\partial t}(q(t)-z I)^{-1}\right\|_{L(E)} \leq \frac{C}{z^{\alpha+1 / 2}},
$$

and

$$
\left\|\frac{\partial}{\partial t}(q(t)-z I)^{-1}-\frac{\partial}{\partial s}(q(s)-z I)^{-1}\right\|_{L(E)} \leq \frac{C|t-s|^{2 \eta}}{z^{\rho+1 / 2}}+\frac{C|t-s|}{z^{2 \alpha}} .
$$


Indeed, the first estimate is a consequence of the identity

$$
\frac{\partial}{\partial t}(q(t)-z I)^{-1}=q(t)(q(t)-z I)^{-1}\left(\frac{d}{d t} q(t)^{-1}\right) q(t)(q(t)-z I)^{-1} .
$$

For the second one, write

$$
\begin{aligned}
\frac{\partial}{\partial t}(q(t)-z I)^{-1}-\frac{\partial}{\partial s}(q(s)- & z I)^{-1} \\
= & q(t)(q(t)-z I)^{-1}\left(\frac{d}{d t} q(t)^{-1}\right) q(t)(q(t)-z I)^{-1} \\
& -q(s)(q(s)-z I)^{-1}\left(\frac{d}{d s} q(s)^{-1}\right) q(s)(q(s)-z I)^{-1} \\
= & q(t)(q(t)-z I)^{-1}\left(\frac{d}{d t} q(t)^{-1}\right) q(t)(q(t)-z I)^{-1} \\
& -q(s)(q(s)-z I)^{-1}\left(\frac{d}{d s} q(s)^{-1}\right) q(t)(q(t)-z I)^{-1} \\
& +q(s)(q(s)-z I)^{-1}\left(\frac{d}{d s} q(s)^{-1}\right) q(t)(q(t)-z I)^{-1} \\
& -q(s)(q(s)-z I)^{-1}\left(\frac{d}{d s} q(s)^{-1}\right) q(s)(q(s)-z I)^{-1} \\
:= & I_{1}-I_{2}+I_{3}-I_{4},
\end{aligned}
$$

thus

$$
\left\|I_{1}-I_{2}\right\| \leq \frac{C|t-s|^{2 \eta}}{z^{\rho+1 / 2}}
$$

and

$$
\begin{aligned}
\left\|I_{3}-I_{4}\right\| & \leq C \frac{z}{z^{\alpha+1 / 2}}\left\|(q(t)-z I)^{-1}-(q(s)-z I)^{-1}\right\| \\
& \leq C \frac{z}{z^{\alpha+1 / 2}}\left\|\int_{s}^{t} \frac{\partial}{\partial r}(q(r)-z I)^{-1} d r\right\| \\
& \leq C \frac{z}{z^{\alpha+1 / 2}} \frac{|t-s|}{z^{\alpha+1 / 2}}=\frac{C|t-s|}{z^{2 \alpha}} .
\end{aligned}
$$

\section{Construction of the solution}

\subsection{Representation of the solution}

In all this paper, for $t \in[0, \delta], z \notin \mathbb{R}_{+}$, we set

$$
c_{\sqrt{-z}}(t)=\frac{\cosh \sqrt{-z}(\delta-t)}{\cosh \sqrt{-z} \delta}, \quad s_{\sqrt{-z}}(t)=\frac{\sinh \sqrt{-z} t}{\cosh \sqrt{-z} \delta} .
$$


Our representation formula can be heuristically derived by the following argument: taking the constant case into account (see formula (4)), we look for a solution of Problem (2) in the following form:

$$
\begin{aligned}
u(t)= & \frac{1}{2 i \pi} \int_{\gamma} c_{\sqrt{-z}}(t)(Q(t)-z I)^{-1} \varphi^{*} d z \\
& +\frac{1}{2 i \pi} \int_{\gamma} s_{\sqrt{-z}}(t)(Q(t)-z I)^{-1} \psi^{*} \frac{d z}{\sqrt{-z}} \\
& -\frac{1}{2 i \pi} \int_{\gamma} \int_{0}^{\delta} K_{\sqrt{-z}}(t, s)(Q(t)-z I)^{-1} f^{*}(s) d s d z, \\
u(t):= & d(t, Q(t)) \varphi^{*}+n(t, Q(t)) \psi^{*}+v\left(t, Q(t), f^{*}\right),
\end{aligned}
$$

The kernel $K_{\sqrt{-z}}$ is defined in (5). We are then concerned with the determination of the triplet $\left(\varphi^{*}, \psi^{*}, f^{*}\right)$ in an adequate space in order that $u$ is a strict solution of Problem (2), when $f \in C^{2 \theta}([0, \delta], E)$, whereas $\varphi$ and $\psi$ are given in some subspaces of $E$.

Remark 1.1. In virtue of (7), it is possible to make use of square roots

$$
-(-Q(t))^{1 / 2}, \quad t \in[0, \delta]
$$

which are well defined and generate analytic semigroups (not strongly continuous at zero), but the calculus are very cumbersome. One must be careful on the use of these fractional powers, since the operators are not densely defined. So, we prefer the natural use of the Green's kernels.

In the sequel, we will use the following notations:

$$
d(t, Q(t)) \varphi^{*}=d(t), \quad n(t, Q(t)) \psi^{*}=n(t), \quad v\left(t, Q(t), f^{*}\right)=v(t) .
$$

Now, let us justify the convergence and the regularity of the integrals occurring in $(11)$.

In this work, $\delta$ is a small fixed positive number.

Our first following fundamental lemma is concerned with the integral

$$
d(t, Q(t)) \varphi^{*}=\frac{1}{2 i \pi} \int_{\gamma} c_{\sqrt{-z}}(t)(Q(t)-z I)^{-1} \varphi^{*} d z .
$$

Lemma 1.2. Under the assumptions (6)-(9), there exists a constant $C$ independent of $\delta$ such that

(i) $\forall t>0, \varphi^{*} \in E, \quad\left\|d(t, Q(t)) \varphi^{*}\right\|_{E} \leq C\left\|\varphi^{*}\right\|_{E}$,

(ii) $\forall t>0, s \geq 0, \varphi^{*} \in E, \quad d(t, Q(s)) \varphi^{*} \in D(Q(s))$ and

$$
\left\|Q(s) d(t, Q(s)) \varphi^{*}\right\|_{E} \leq\left(C / t^{2}\right)\left\|\varphi^{*}\right\|_{E} .
$$


(iii) $\forall \varphi^{*} \in D(Q(0)), t>0, s \in[0, t]$,

$$
d(t, Q(s)) \varphi^{*} \in D(Q(s)),
$$

and

$$
\left\|Q(s) d(t, Q(s)) \varphi^{*}\right\|_{E} \leq C\left\|Q(0) \varphi^{*}\right\|_{E} .
$$

In particular

$$
\left\|Q(t) d(t, Q(t)) \varphi^{*}\right\|_{E} \leq\left\|Q(0) \varphi^{*}\right\|_{E} .
$$

(iv) One has

$$
\lim _{t \rightarrow 0} d(t, Q(t)) \varphi^{*}=\varphi^{*} \quad \text { iff } \quad \varphi^{*} \in \overline{D(Q(0))}
$$

and

$$
t \mapsto d(t, Q(0)) \varphi^{*} \in C^{2 \theta}([0, \delta], E)
$$

if and only if

$$
\varphi^{*} \in D_{Q(0)}(\theta,+\infty) .
$$

(For this interpolation space, see Grisvard [7, p. 664].)

Proof. Assertions (i) and (ii). They can be treated similarly. So, we restrict ourselves to the second one. For $z \in \gamma,|z| \geq r_{0}, \delta>0$, and $t>0$, we have

$$
\left\{\begin{array}{l}
\operatorname{Re}(\sqrt{-z} \delta)>0 \text { and } \operatorname{Re}(\sqrt{-z} t)>0 \\
\arg (\sqrt{-z} \delta)=\arg (\sqrt{-z} t)=\pi / 2-\theta_{0} / 2=\theta_{1}>0 \\
\operatorname{Re}(\sqrt{-z} \delta)=\delta|z|^{1 / 2} \cos \left(\pi / 2-\theta_{0} / 2\right)=\delta|z|^{1 / 2} \sin \left(\theta_{0} / 2\right) .
\end{array}\right.
$$

Hence

$$
\left|1+e^{-2 \sqrt{-z} \delta}\right|^{2}=1+2 e^{-2 \operatorname{Re} \sqrt{-z} \delta} \cos (2 \operatorname{Im} \sqrt{-z} \delta)+e^{-4 \operatorname{Re} \sqrt{-z} \delta},
$$

and then, if $\cos (2 \operatorname{Im} \sqrt{-z} \delta) \geq 0$,

$$
\left|1+e^{-2 \sqrt{-z} \delta}\right|^{2} \geq 1,
$$

whereas if $\cos (2 \operatorname{Im} \sqrt{-z} \delta) \leq 0$, i.e., if $2 \operatorname{Re}(\sqrt{-z} \delta) \geq \pi /\left(2 \tan \theta_{1}\right)$

$$
\begin{aligned}
\left|1+e^{-2 \sqrt{-z} \delta}\right|^{2} & \geq 1-2 e^{-2 \operatorname{Re}(\sqrt{-z} \delta)}+e^{-4 \operatorname{Re}(\sqrt{-z} \delta)} \\
& \left.=\left(1-e^{-2 \operatorname{Re}(\sqrt{-z} \delta)}\right)^{2} \geq\left(1-e^{-\pi /\left(2 \tan \theta_{1}\right.}\right)\right)^{2}=C\left(\theta_{0}\right)>0 .
\end{aligned}
$$

Therefore, we obtain in both cases

$$
\begin{aligned}
\left|c_{\sqrt{-z}}(t)\right| & =\left|\frac{e^{\sqrt{-z}(\delta-t)}+e^{-\sqrt{-z}(\delta-t)}}{e^{\sqrt{-z} \delta}+e^{-\sqrt{-z} \delta}}\right|=\left|e^{-\sqrt{-z} t}\right|\left|\frac{1+e^{-2 \sqrt{-z}(\delta-t)}}{1+e^{-2 \sqrt{-z} \delta}}\right| \\
& \leq C e^{-\operatorname{Re}(\sqrt{-z}) t} \leq C e^{-t|z|^{1 / 2}} \sin \left(\theta_{0} / 2\right)
\end{aligned}
$$


where $C$ is independent of $\delta, t$, and $z$. From this estimate it follows that $\forall \varphi^{*} \in E$, $\forall t>0$, the integral $d(t, Q(t)) \varphi^{*}$ is absolutely convergent and

$$
\begin{aligned}
d(t, Q(t)) \varphi^{*}= & \frac{1}{2 i \pi} \int_{\gamma,|z| \geq \frac{1}{t^{2}}} c_{\sqrt{-z}}(t)(Q(t)-z I)^{-1} \varphi^{*} d z \\
& +\frac{1}{2 i \pi} \int_{\gamma,|z| \leq \frac{1}{t^{2}}} c_{\sqrt{-z}}(t)(Q(t)-z I)^{-1} \varphi^{*} d z \\
d(t, Q(t)) \varphi^{*}:= & I_{1}+I_{2} .
\end{aligned}
$$

Then

$$
\begin{aligned}
\left\|I_{1}\right\| & \leq C\left(\int_{\gamma,|z| \geq \frac{1}{t^{2}}} \frac{e^{-t|z|^{1 / 2} \sin \left(\theta_{0} / 2\right)}}{|z|}|d z|\right)\left\|\varphi^{*}\right\|_{E} \\
& \leq C\left(\int_{1}^{+\infty} \frac{e^{-\sigma}}{\sigma} d \sigma\right)\left\|\varphi^{*}\right\|_{E} \\
& \leq C\left\|\varphi^{*}\right\|_{E} .
\end{aligned}
$$

Concerning the integral $I_{2}$, write

$$
\begin{aligned}
I_{2}= & \frac{1}{2 i \pi} \int_{\gamma,|z| \leq \frac{1}{t^{2}}}\left(c_{\sqrt{-z}}(t)-1\right)(Q(t)-z I)^{-1} \varphi^{*} d z \\
& +\frac{1}{2 i \pi} \int_{\gamma,|z| \leq \frac{1}{t^{2}}}(Q(t)-z I)^{-1} \varphi^{*} d z, \\
I_{2}:= & I_{2}^{\prime}+I_{2}^{\prime \prime} .
\end{aligned}
$$

Consequently,

$$
\begin{aligned}
I_{2}^{\prime \prime} & =\frac{1}{2 i \pi} \int_{C_{t}}(Q(t)-z I)^{-1} \varphi^{*} d z-\frac{1}{2 i \pi} \int_{\Gamma_{t}}(Q(t)-z I)^{-1} \varphi^{*} d z \\
& =-\frac{1}{2 i \pi} \int_{\Gamma_{t}}(Q(t)-z I)^{-1} \varphi^{*} d z .
\end{aligned}
$$

where

$$
C_{t}=\left\{z \in \gamma:|z| \leq 1 / t^{2}\right\} \cup \Gamma_{t}, \quad \Gamma_{t}=\left\{z=e^{i \tau} / t^{2}: \tau \in\left[-\theta_{0}, \theta_{0}\right]\right\} .
$$

But

$$
\left\|-\frac{1}{2 i \pi} \int_{\Gamma_{t}}(Q(t)-z I)^{-1} \varphi^{*} d z\right\| \leq C\left\|\varphi^{*}\right\|
$$


and

$$
\begin{aligned}
I_{2}^{\prime} & =\frac{1}{2 i \pi} \int_{\gamma,|z| \leq \frac{1}{t^{2}}}\left(c_{\sqrt{-z}}(t)-1\right)(Q(t)-z I)^{-1} \varphi^{*} d z \\
& =\frac{1}{2 i \pi} \int_{\gamma,|z| \leq \frac{1}{t^{2}}} \sqrt{-z}\left(\int_{0}^{t} s_{\sqrt{-z}}(\xi-\delta) d \xi\right)(Q(t)-z I)^{-1} \varphi^{*} d z, \\
\left\|I_{2}^{\prime}\right\| & \leq C \int_{r_{0}}^{\frac{1}{t^{2}}} t|z|^{-1 / 2}\left\|\varphi^{*}\right\| d|z| \leq C\left\|\varphi^{*}\right\| .
\end{aligned}
$$

The same arguments apply for the second statement concerning

$$
Q(s) d(t, Q(s)) \varphi^{*}=\frac{1}{2 i \pi} \int_{\gamma} c_{\sqrt{-z}}(t) Q(s)(Q(s)-z I)^{-1} \varphi^{*} d z .
$$

Assertion (iii). Suppose that $\varphi^{*} \in D(Q(0)), t>0$ and $s \in[0, t]$. We have

$$
\begin{aligned}
Q(s) d(t, Q(s)) \varphi^{*}= & \frac{1}{2 i \pi} \int_{\gamma} c_{\sqrt{-z}}(t) Q(s)(Q(s)-z I)^{-1} \varphi^{*} d z \\
= & \frac{1}{2 i \pi} \int_{\gamma} c_{\sqrt{-z}}(t) Q(s)(Q(s)-z I)^{-1}\left[Q(0)^{-1}-Q(s)^{-1}\right] Q(0) \varphi^{*} d z \\
& +\frac{1}{2 i \pi} \int_{\gamma} c_{\sqrt{-z}}(t)(Q(s)-z I)^{-1} Q(0) \varphi^{*} d z .
\end{aligned}
$$

As in the first statement, we get

$$
\left\|\frac{1}{2 i \pi} \int_{\gamma} c_{\sqrt{-z}}(t)(Q(s)-z I)^{-1} Q(0) \varphi^{*} d z\right\| \leq C\left\|Q(0) \varphi^{*}\right\|_{E} .
$$

Regarding the first integral, we have

$$
\begin{aligned}
& \frac{1}{2 i \pi} \int_{\gamma} c_{\sqrt{-z}}(t) Q(s)(Q(s)-z I)^{-1}\left[Q(0)^{-1}-Q(s)^{-1}\right] Q(0) \varphi^{*} d z \\
& =\frac{1}{2 i \pi} \int_{\gamma} c_{\sqrt{-z}}(t) Q(s)(Q(s)-z I)^{-1}\left(Q(0)^{-1}-Q(s)^{-1}+s \frac{d}{d s} Q(s)_{\mid s=0}^{-1}\right) Q(0) \varphi^{*} d z \\
& \quad+\frac{1}{2 i \pi} \int_{\gamma} c_{\sqrt{-z}}(t) s Q(s)(Q(s)-z I)^{-1}\left(\frac{d}{d s} Q(s)^{-1}-\frac{d}{d s} Q(s)_{\mid s=0}^{-1}\right) Q(0) \varphi^{*} d z \\
& \quad-\frac{1}{2 i \pi} \int_{\gamma} c_{\sqrt{-z}}(t) s Q(s)(Q(s)-z I)^{-1}\left(\frac{d}{d s} Q(s)^{-1}\right) Q(0) \varphi^{*} d z \\
& :=J_{1}+J_{2}+J_{3} .
\end{aligned}
$$


By the mean value theorem we derive the following estimate of $J_{1}$ :

$$
\begin{aligned}
\| Q(0)^{-1}-Q(s)^{-1}+ & s \frac{d}{d s} Q(s)_{\mid s=0}^{-1} \| \\
& =\left\|-\int_{0}^{s}\left(\frac{d}{d \xi} Q(\xi)^{-1}-\frac{d}{d s} Q(s)_{\mid s=0}^{-1}\right) d \xi\right\| \\
& \leq \int_{0}^{s}|\xi| \sup _{\theta \in] 0,1[}\left\|\frac{d^{2}}{d s^{2}} Q(s)_{\mid s=\theta \xi}^{-1}\right\| d \xi \leq s^{2} \sup _{\theta \in] 0,1[}\left\|\frac{d^{2}}{d s^{2}} Q(s)_{\mid s=\theta \xi}^{-1}\right\| \\
& \leq t^{2} \sup _{\theta \in] 0,1[}\left\|\frac{d^{2}}{d s^{2}} Q(s)_{\mid s=\theta \xi}^{-1}\right\|,
\end{aligned}
$$

which leads to

$$
\left\|J_{1}\right\| \leq C\left\|Q(0) \varphi^{*}\right\| .
$$

For $J_{2}$, we have

$$
\begin{gathered}
\left\|s Q(s)(Q(s)-z I)^{-1}\left(\frac{d}{d s} Q(s)^{-1}-\frac{d}{d s} Q(s)_{\mid s=0}^{-1}\right) Q(0) \varphi^{*}\right\| \\
\quad=\left\|s Q(s)(Q(s)-z I)^{-1}\left(\int_{0}^{s} \frac{d^{2}}{d r^{2}} Q(r)^{-1} d r\right) Q(0) \varphi^{*}\right\| \\
\leq s^{2} \sup _{s \in(0,1)}\left\|\frac{d^{2}}{d s^{2}} Q(s)^{-1}\right\|_{L(E)},
\end{gathered}
$$

and we apply a similar treatment as in the previous assertion. Concerning the integral $J_{3}$, note that

$$
\begin{aligned}
\left(\int_{\gamma,|z| \geq \frac{1}{t^{2}}} \frac{s e^{-t|z|^{1 / 2} \sin \left(\theta_{0} / 2\right)}}{|z|^{\alpha+1 / 2}}|d z|\right)\left\|Q(0) \varphi^{*}\right\|_{E} & \\
& \leq C\left(\int_{1}^{\infty} \frac{2 \sigma s e^{-\sigma} d \sigma}{t^{2}\left(\frac{\sigma}{t}\right)^{2 \alpha+1}}\right)\left\|Q(0) \varphi^{*}\right\|_{E} \\
& \leq C s t^{2 \alpha-1}\left\|Q(0) \varphi^{*}\right\|_{E} \leq C t^{2 \alpha}\left\|Q(0) \varphi^{*}\right\|_{E},
\end{aligned}
$$

and a similar estimate holds true on the part $\left\{z \in \gamma,|z| \leq \frac{1}{t^{2}}\right\}$ of the curve $\gamma$. This yields

$$
\left\|J_{3}\right\| \leq C\left\|Q(0) \varphi^{*}\right\| .
$$

Assertion (iv). For all $\varphi^{*} \in E$, we have

$$
\begin{aligned}
d(t, Q(t)) \varphi^{*} & \left.=\frac{1}{2 i \pi} \int_{\gamma} c_{\sqrt{-z}}(t)(Q(t)-z I)^{-1}-(Q(0)-z I)^{-1}\right) \varphi^{*} d z \\
& +\frac{1}{2 i \pi} \int_{\gamma} c_{\sqrt{-z}}(t)(Q(0)-z I)^{-1} \varphi^{*} d z=a(t)+b(t) .
\end{aligned}
$$


We can show by the Dunford's calculus that the term

$$
b(t):=\frac{1}{2 i \pi} \int_{\gamma} c_{\sqrt{-z}}(t)(Q(0)-z I)^{-1} \varphi^{*} d z
$$

can be rewritten as follows:

$$
b(t)=\left(I+e^{-2 \delta(-Q(0))^{1 / 2}}\right)^{-1}\left(I+e^{-2(-Q(0))^{1 / 2}(\delta-t)}\right) e^{-(-Q(0))^{1 / 2} t} \varphi^{*},
$$

where $\left(e^{-(-Q(0))^{1 / 2} t}\right)_{t>0}$ is the analytic semigroup (not strongly continuous at zero) associated with $-(-Q(0))^{1 / 2}$. Thanks to Lunardi [10, p. 59], the operator

$$
\left(I+e^{-2 \delta(-Q(0))^{1 / 2}}\right)^{-1}
$$

is well defined. By Sinestrari [11, Proposition 1.2, p. 20], we deduce that

$$
b(t) \rightarrow \varphi^{*}, \quad \text { as } t \rightarrow 0
$$

if and only if $\varphi^{*} \in \overline{D\left((-Q(0))^{1 / 2}\right)}=\overline{D(Q(0))}$. This last equality follows from the properties of fractional powers of sectorial operators, see Haase [8].

Regarding the term $a(t)$, we have the estimate

$$
\begin{aligned}
\| \int_{\gamma} c_{\sqrt{-z}}(t)(Q(t)-z I)^{-1}- & \left.(Q(0)-z I)^{-1}\right) \varphi^{*} d z \| \\
= & \left\|\int_{\gamma} c_{\sqrt{-z}}(t) \int_{0}^{t} \frac{\partial}{\partial r}(Q(r)-z I)^{-1} \varphi^{*} d r d z\right\| \\
\leq & \left\|\int_{\gamma,|z| \geq \frac{1}{t^{2}}} c_{\sqrt{-z}}(t) \int_{0}^{t} \frac{\partial}{\partial r}(Q(r)-z I)^{-1} \varphi^{*} d r d z\right\| \\
& +\left\|\int_{\gamma,|z| \leq \frac{1}{t^{2}}} c_{\sqrt{-z}}(t) \int_{0}^{t} \frac{\partial}{\partial r}(Q(r)-z I)^{-1} \varphi^{*} d r d z\right\| \\
:= & a_{1}(t)+a_{2}(t)
\end{aligned}
$$

with

$$
\begin{aligned}
a_{1}(t) & \leq C\left(\int_{\gamma,|z| \geq \frac{1}{t^{2}}} \frac{t e^{-t|z|^{1 / 2} \sin \left(\theta_{0} / 2\right)}}{|z|^{\alpha+1 / 2}}|d z|\right)\left\|\varphi^{*}\right\|_{E} \\
& \leq C\left(\int_{\gamma,|z| \geq \frac{1}{t^{2}}} \frac{t e^{-t|z|^{1 / 2} \sin \left(\theta_{0} / 2\right)}}{\left.l v e r t z\right|^{\alpha+1 / 2}}|d z|\right)\left\|\varphi^{*}\right\|_{E} \\
& \leq C t^{2 \alpha}\left\|\varphi^{*}\right\|_{E},
\end{aligned}
$$


and

$$
\begin{aligned}
a_{2}(t) \leq & \left\|\int_{\gamma,|z| \leq \frac{1}{t^{2}}}\left(c_{\sqrt{-z}}(t)-1\right) \int_{0}^{t} \frac{\partial}{\partial r}(Q(r)-z I)^{-1} \varphi^{*} d r d z\right\| \\
& +\left\|\int_{\gamma,|z| \leq \frac{1}{t^{2}}} \int_{0}^{t} \frac{\partial}{\partial r}(Q(r)-z I)^{-1} \varphi^{*} d r d z\right\| \\
a_{2}(t):= & a_{21}(t)+a_{22}(t) .
\end{aligned}
$$

Concerning the first term $a_{21}(t)$, we observe that

$$
a_{21}(t) \leq C\left(\int_{\gamma,|z| \leq \frac{1}{t^{2}}} \frac{t|z|^{1 / 2} t}{|z|^{\alpha+1 / 2}}|d z|\right)\left\|\varphi^{*}\right\|_{E} \leq C t^{2 \alpha}\left\|\varphi^{*}\right\|_{E} .
$$

For the second one, $a_{22}(t)$, we close the curve at the right-hand side and we denote by $C_{t}$ the arc

$$
\left(1 / t^{2}\right) e^{i \theta}, \quad \theta \in\left[-\theta_{0},+\theta_{0}\right]
$$

to obtain

$$
\begin{aligned}
a_{22}(t) & =\left\|-\int_{C_{t}} \int_{0}^{t} \frac{\partial}{\partial r}(Q(r)-z I)^{-1} \varphi^{*} d r d z\right\| \\
& \leq \int_{-\theta_{0}}^{\theta_{0}}\left\|\int_{0}^{t} \frac{\partial}{\partial r}\left(Q(r)-\left(e^{i \theta} / t^{2}\right) I\right)^{-1} \varphi^{*} d r\right\| \frac{d \theta}{t^{2}} \\
& \leq C t^{2 \alpha}\left\|\varphi^{*}\right\|_{E},
\end{aligned}
$$

The second property is proved in Sinestrari [11, Proposition 1.2, p. 20].

This ends the proof of the convergence $d(t, Q(t)) \varphi^{*} \rightarrow \varphi^{*}$ as $t \rightarrow 0$. Observe that it is possible to obtain the same result using a direct calculus as in Labbas [9].

Remark 1.3. In order to simplify the calculus, we will often consider that $c_{0}=1$.

Proposition 1.4. Suppose that $\left.f^{*} \in C^{\beta}([0, \delta], E), \beta \in\right] 0,1[$. Then, under the assumptions (6)-(9), the function $v$ (introduced in section 1.1) defined by

$$
v(t)=-\frac{1}{2 i \pi} \int_{\gamma} \int_{0}^{\delta} K_{\sqrt{-z}}(t, s)(Q(t)-z I)^{-1} f^{*}(s) d s d z,
$$

(the kernel $K_{\sqrt{-z}}$ is defined in (5)) enjoys the properties:

(i) $v(\cdot) \in C^{2}(] 0, \delta[; E)$, 
(ii) $\forall t \in] 0, \delta\left[v^{\prime \prime}(t)+p(t) v^{\prime}(t)+Q(t) v(t)=f^{*}(t)+P_{\lambda}\left(f^{*}\right)(t)+R_{\lambda}\left(f^{*}\right)(t)\right.$ where

$$
\begin{aligned}
P_{\lambda}\left(f^{*}\right)(t)= & -\frac{1}{2 i \pi} \int_{\gamma} \int_{0}^{\delta} K_{\sqrt{-z}}(t, s) \frac{\partial^{2}}{\partial t^{2}}(Q(t)-z I)^{-1} f^{*}(s) d s d z \\
& +\frac{1}{i \pi} \int_{\gamma} \int_{0}^{\delta} \frac{\partial}{\partial t} K_{\sqrt{-z}}(t, s) \frac{\partial}{\partial t}(Q(t)-z I)^{-1} f^{*}(s) d s d z,
\end{aligned}
$$

and

$$
\begin{aligned}
R_{\lambda}\left(f^{*}\right)(t)= & -\frac{1}{2 i \pi} \int_{\gamma} \int_{0}^{\delta} K_{\sqrt{-z}}(t, s) p(t) \frac{\partial}{\partial t}(Q(t)-z I)^{-1} f^{*}(s) d s d z \\
& -\frac{1}{2 i \pi} \int_{\gamma} \int_{0}^{\delta} \frac{\partial}{\partial t} K_{\sqrt{-z}}(t, s) p(t)(Q(t)-z I)^{-1} f^{*}(s) d s d z .
\end{aligned}
$$

Proof. Assertion (i). Step 1. Using a direct computation on the kernel (5), one obtains the existence of some constant depending only on $\theta_{0}$ such that for all $z \in \gamma$

$$
\begin{aligned}
\left\|\int_{0}^{\delta} K_{\sqrt{-z}}(t, s) f^{*}(s) d s\right\| & \leq\left(\sup _{t \in[0,1]} \int_{0}^{\delta}\left|K_{\sqrt{-z}}(t, s)\right| d s\right)\left\|f^{*}\right\|_{C(E)} \\
& \leq \frac{\left\|f^{*}\right\|_{C(E)}}{|z| \cos \left(\pi / 2-\theta_{0} / 2\right)}=\frac{C\left\|f^{*}\right\|_{C(E)}}{|z|}
\end{aligned}
$$

(see [6, p. 372]) which implies that the integral

$$
v(t)=-\frac{1}{2 i \pi} \int_{\gamma} \int_{0}^{\delta} K_{\sqrt{-z}}(t, s)(Q(t)-z I)^{-1} f^{*}(s) d s d z,
$$

converges. But it is not possible to apply $Q(t)$ on this representation. One must use the Holderianity of $f^{*}$. So, we write $v$ as follows:

$$
\begin{aligned}
v(t)= & -\frac{1}{2 i \pi} \int_{\gamma} \int_{0}^{\delta} K_{\sqrt{-z}}(t, s)(Q(t)-z I)^{-1}\left(f^{*}(s)-f^{*}(t)\right) d s d z \\
& -\frac{1}{2 i \pi} \int_{\gamma} \int_{0}^{\delta} K_{\sqrt{-z}}(t, s)(Q(t)-z I)^{-1} f^{*}(t) d s d z \\
= & -\frac{1}{2 i \pi} \int_{\gamma} \int_{0}^{\delta} K_{\sqrt{-z}}(t, s)(Q(t)-z I)^{-1}\left(f^{*}(s)-f^{*}(t)\right) d s d z \\
& +\frac{1}{2 i \pi} \int_{\gamma} \frac{(\cosh \sqrt{-z} t-1) \cosh \sqrt{-z}(\delta-t)}{z \cosh \sqrt{-z} \delta}(Q(t)-z I)^{-1} f^{*}(t) d z \\
& +\frac{1}{2 i \pi} \int_{\gamma} \frac{\sinh \sqrt{-z} t \sinh \sqrt{-z}(\delta-t)}{z \cosh \sqrt{-z} \delta}(Q(t)-z I)^{-1} f^{*}(t) d z,
\end{aligned}
$$


which becomes

$$
\begin{aligned}
v(t)= & -\frac{1}{2 i \pi} \int_{\gamma} \int_{0}^{\delta} K_{\sqrt{-z}}(t, s)(Q(t)-z I)^{-1}\left(f^{*}(s)-f^{*}(t)\right) d s d z \\
& +\frac{1}{2 i \pi} \int_{\gamma} \frac{(Q(t)-z I)^{-1} f^{*}(t)}{z} d z \\
& -\frac{1}{2 i \pi} \int_{\gamma} c_{\sqrt{-z}}(t) \frac{(Q(t)-z I)^{-1}}{z} f^{*}(t) d z .
\end{aligned}
$$

Step 2. By the Cauchy formula, we get

$$
\begin{aligned}
v(t)= & -\frac{1}{2 i \pi} \int_{\gamma} \int_{0}^{\delta} K_{\sqrt{-z}}(t, s)(Q(t)-z I)^{-1}\left(f^{*}(s)-f^{*}(t)\right) d s d z \\
& -\frac{1}{2 i \pi} \int_{\gamma} c_{\sqrt{-z}}(t) \frac{(Q(t)-z I)^{-1}}{z} f^{*}(t) d z \\
& +Q(t)^{-1} f^{*}(t) .
\end{aligned}
$$

Since $f^{*}$ is Hölderian of exponent $\left.\beta \in\right] 0,1[$, then

$$
\begin{aligned}
\| \frac{1}{2 i \pi} \int_{\gamma} \int_{0}^{\delta} K_{\sqrt{-z}}(t, s)(Q(t)- & z I)^{-1}\left(f^{*}(s)-f^{*}(t)\right) d s d z \| \\
& \leq \int_{\gamma} \frac{C}{|z|} \int_{0}^{\delta}\left|K_{\sqrt{-z}}(t, s)\right||t-s|^{\beta}\left\|f^{*}\right\|_{C^{\beta}(E)} d s|d z| \\
& \leq C\left\|f^{*}\right\|_{C^{\beta}(E)} \int_{\gamma} \frac{|d z|}{|z|^{2+\beta / 2}} \leq C\left\|f^{*}\right\|_{C^{\beta}(E)},
\end{aligned}
$$

(see [6, p. 376]) and

$$
\begin{aligned}
\| \frac{1}{2 i \pi} \int_{\gamma} \int_{0}^{\delta} K_{\sqrt{-z}}(t, s) Q(t)(Q(t) & -z I)^{-1}\left(f^{*}(s)-f^{*}(t)\right) d s d z \| \\
& \leq \int_{\gamma} \frac{C}{|z|^{1+\beta / 2}}|d z|\left\|f^{*}\right\|_{C^{\beta}(E)} \leq C\left\|f^{*}\right\|_{C^{\beta}(E)} .
\end{aligned}
$$

Consequently

$$
\forall t \in] 0, \delta[, \quad v(t) \in D(Q(t))
$$

because (as in the proof of Lemma 1.2)

$$
\left\|\frac{1}{2 i \pi} \int_{\gamma} c_{\sqrt{-z}}(t) \frac{Q(t)(Q(t)-z I)^{-1}}{z} f^{*}(t) d z\right\| \leq C\left\|f^{*}\right\|_{C(E)} .
$$


(The factor $1 / t^{2}$ is compensated by $1 /|z|$.) Hence

$$
\begin{aligned}
Q(t) v(t)= & -\frac{1}{2 i \pi} \int_{\gamma} \int_{0}^{\delta} K_{\sqrt{-z}}(t, s) Q(t)(Q(t)-z I)^{-1}\left(f^{*}(s)-f^{*}(t)\right) d s d z \\
& -\frac{1}{2 i \pi} \int_{\gamma} c_{\sqrt{-z}}(t) \frac{Q(t)(Q(t)-z I)^{-1}}{z} f^{*}(t) d z+f^{*}(t) .
\end{aligned}
$$

Assertion (ii). Step 1. Regarding the derivative $v^{\prime}(t)$, we have

$$
\begin{aligned}
v^{\prime}(t)= & \frac{1}{2 i \pi} \int_{\gamma} \int_{0}^{t} \frac{\sinh \sqrt{-z} s \sinh \sqrt{-z}(\delta-t)}{\cosh \sqrt{-z} \delta}(Q(t)-z I)^{-1} f^{*}(s) d s d z \\
& -\frac{1}{2 i \pi} \int_{\gamma} \int_{t}^{\delta} \frac{\cosh \sqrt{-z} t \cosh \sqrt{-z}(\delta-s)}{\cosh \sqrt{-z} \delta}(Q(t)-z I)^{-1} f^{*}(s) d s d z \\
& -\frac{1}{2 i \pi} \int_{\gamma} \int_{0}^{t} \frac{\sinh \sqrt{-z} s \cosh \sqrt{-z}(t-\delta)}{\sqrt{-z} \cosh \sqrt{-z} \delta} \frac{\partial}{\partial t}(Q(t)-z I)^{-1} f^{*}(s) d s d z \\
& -\frac{1}{2 i \pi} \int_{\gamma} \int_{t}^{\delta} \frac{\sinh \sqrt{-z} t \cosh \sqrt{-z}(s-\delta)}{\sqrt{-z} \cosh \sqrt{-z} \delta} \frac{\partial}{\partial t}(Q(t)-z I)^{-1} f^{*}(s) d s d z .
\end{aligned}
$$

By Assumption (6), it is easy to see that

$$
\begin{aligned}
p(t) v^{\prime}(t)= & \frac{1}{2 i \pi} \int_{\gamma} \int_{0}^{t} \frac{\sinh \sqrt{-z} s \sinh \sqrt{-z}(\delta-t)}{\cosh \sqrt{-z} \delta} p(t)(Q(t)-z I)^{-1} f^{*}(s) d s d z \\
& -\frac{1}{2 i \pi} \int_{\gamma} \int_{t}^{\delta} \frac{\cosh \sqrt{-z} t \cosh \sqrt{-z}(\delta-s)}{\cosh \sqrt{-z} \delta} p(t)(Q(t)-z I)^{-1} f^{*}(s) d s d z \\
& -\frac{1}{2 i \pi} \int_{\gamma} \int_{0}^{t} \frac{\sinh \sqrt{-z} s \cosh \sqrt{-z}(t-\delta)}{\sqrt{-z} \cosh \sqrt{-z} \delta} p(t) \frac{\partial}{\partial t}(Q(t)-z I)^{-1} f^{*}(s) d s d z \\
& -\frac{1}{2 i \pi} \int_{\gamma} \int_{t}^{\delta} \frac{\sinh \sqrt{-z} t \cosh \sqrt{-z}(s-\delta)}{\sqrt{-z} \cosh \sqrt{-z} \delta} p(t) \frac{\partial}{\partial t}(Q(t)-z I)^{-1} f^{*}(s) d s d z .
\end{aligned}
$$

Therefore

$$
\begin{aligned}
p(t) v^{\prime}(t)= & -\frac{1}{2 i \pi} \int_{\gamma} \int_{0}^{\delta} K_{\sqrt{-z}}(t, s) p(t) \frac{\partial}{\partial t}(Q(t)-z I)^{-1} f^{*}(s) d s d z \\
& -\frac{1}{2 i \pi} \int_{\gamma} \int_{0}^{\delta} \frac{\partial}{\partial t} K_{\sqrt{-z}}(t, s) p(t)(Q(t)-z I)^{-1} f^{*}(s) d s d z \\
:= & R_{\lambda}\left(f^{*}\right)(t) .
\end{aligned}
$$


Step 2. Let us study the second derivative $v^{\prime \prime}(t)$. The calculus requires some details. We use the method delivered to prove Theorem 3.3.4 in [12, p. 70]. Let $\varepsilon$ be a very small positive number and $t$ be such that

$$
0<\varepsilon \leq t \leq \delta-\varepsilon<\delta
$$

and $v_{\varepsilon}^{\prime}$ be the function defined by

$$
\begin{aligned}
v_{\varepsilon}^{\prime}(t)= & \frac{1}{2 i \pi} \int_{\gamma} \int_{0}^{t-\varepsilon} \frac{\sinh \sqrt{-z} s \sinh \sqrt{-z}(\delta-t)}{\cosh \sqrt{-z} \delta}(Q(t)-z I)^{-1} f^{*}(s) d s d z \\
& -\frac{1}{2 i \pi} \int_{\gamma} \int_{t+\varepsilon}^{\delta} \frac{\cosh \sqrt{-z} s \cosh \sqrt{-z}(\delta-t)}{\cosh \sqrt{-z} \delta}(Q(t)-z I)^{-1} f^{*}(s) d s d z \\
& -\frac{1}{2 i \pi} \int_{\gamma} \int_{0}^{t} \frac{\sinh \sqrt{-z} s \cosh \sqrt{-z}(\delta-t)}{\sqrt{-z} \cosh \sqrt{-z} \delta} \frac{\partial}{\partial t}(Q(t)-z I)^{-1} f^{*}(s) d s d z \\
& -\frac{1}{2 i \pi} \int_{\gamma} \int_{t}^{\delta} \frac{\sinh \sqrt{-z} t \cosh \sqrt{-z}(s-\delta)}{\sqrt{-z} \cosh \sqrt{-z} \delta} \frac{\partial}{\partial t}(Q(t)-z I)^{-1} f^{*}(s) d s d z
\end{aligned}
$$

It is not difficult to see that all these integrals are absolutely convergent and $v_{\varepsilon}^{\prime}(t) \rightarrow v^{\prime}(t)$ strongly as $\varepsilon \rightarrow 0$. In addition, we have

$$
v_{\varepsilon}^{\prime \prime}(t):=V_{\varepsilon}^{1}(t)+V_{\varepsilon}^{2}(t)+V_{\varepsilon}^{3}(t)+V^{4}(t)
$$

where

$$
\begin{aligned}
V_{\varepsilon}^{1}(t)= & \frac{1}{2 i \pi} \int_{\gamma} \int_{0}^{t-\varepsilon} \frac{\sinh \sqrt{-z} s \sinh \sqrt{-z}(\delta-t)}{\cosh \sqrt{-z} \delta} \frac{\partial}{\partial t}(Q(t)-z I)^{-1} f^{*}(s) d s d z \\
& -\frac{1}{2 i \pi} \int_{\gamma} \int_{t+\varepsilon}^{\delta} \frac{\cosh \sqrt{-z} t \cosh \sqrt{-z}(\delta-s)}{\cosh \sqrt{-z} \delta} \frac{\partial}{\partial t}(Q(t)-z I)^{-1} f^{*}(s) d s d z, \\
V_{\varepsilon}^{2}(t)= & \frac{-1}{2 i \pi} \int_{\gamma} \int_{0}^{t-\varepsilon} \frac{\sqrt{-z} \sinh \sqrt{-z} s \cosh \sqrt{-z}(\delta-t)}{\cosh \sqrt{-z} \delta}(Q(t)-z I)^{-1} f^{*}(s) d s d z \\
& -\frac{1}{2 i \pi} \int_{\gamma} \int_{t+\varepsilon}^{\delta} \frac{\sqrt{-z} \sinh \sqrt{-z} t \cosh \sqrt{-z}(\delta-s)}{\cosh \sqrt{-z} \delta}(Q(t)-z I)^{-1} f^{*}(s) d s d z, \\
V_{\varepsilon}^{3}(t)= & \frac{1}{2 i \pi} \int_{\gamma} \frac{\sinh \sqrt{-z}(t-\varepsilon) \sinh \sqrt{-z}(\delta-t)}{\cosh \sqrt{-z} \delta}(Q(t)-z I)^{-1} f^{*}(t-\varepsilon) d z \\
& +\frac{1}{2 i \pi} \int_{\gamma} \frac{\cosh \sqrt{-z} t \cosh \sqrt{-z}(\delta-t-\varepsilon)}{\cosh \sqrt{-z} \delta}(Q(t)-z I)^{-1} f^{*}(t+\varepsilon) d z
\end{aligned}
$$




$$
\begin{aligned}
& V^{4}(t)=-\frac{1}{2 i \pi} \int_{\gamma} \int_{0}^{\delta} K_{\sqrt{-z}}(t, s) \frac{\partial^{2}}{\partial t^{2}}(Q(t)-z I)^{-1} f^{*}(s) d s d z \\
& +\frac{1}{2 i \pi} \int_{\gamma} \int_{0}^{t} \frac{\sinh \sqrt{-z} s \sinh \sqrt{-z}(\delta-t)}{\cosh \sqrt{-z} \delta} \frac{\partial}{\partial t}(Q(t)-z I)^{-1} f^{*}(s) d s d z \\
& -\frac{1}{2 i \pi} \int_{\gamma} \int_{t}^{\delta} \frac{\cosh \sqrt{-z} t \cosh \sqrt{-z}(\delta-s)}{\cosh \sqrt{-z} \delta} \frac{\partial}{\partial t}(Q(t)-z I)^{-1} f^{*}(s) d s d z \text {, }
\end{aligned}
$$

Step 3. We transform $V_{\varepsilon}^{2}(t)$ using the relation

$$
\sqrt{-z}(Q(t)-z I)^{-1}=\frac{-z}{\sqrt{-z}}(Q(t)-z I)^{-1}=\frac{I}{\sqrt{-z}}-\frac{Q(t)(Q(t)-z I)^{-1}}{\sqrt{-z}} .
$$

Then the Dunford calculus and the Hölderian regularity of $f^{*}$ yield

$$
\begin{aligned}
V_{\varepsilon}^{2}(t)= & \frac{1}{2 i \pi} \int_{\gamma} \int_{0}^{t-\varepsilon} \frac{\sinh \sqrt{-z} s \cosh \sqrt{-z}(\delta-t)}{\sqrt{-z} \cosh \sqrt{-z} \delta} Q(t)(Q(t)-z I)^{-1} f^{*}(s) d s d z \\
& +\frac{1}{2 i \pi} \int_{\gamma} \int_{t+\varepsilon}^{\delta} \frac{\sinh \sqrt{-z} t \cosh \sqrt{-z}(\delta-s)}{\sqrt{-z} \cosh \sqrt{-z} \delta} Q(t)(Q(t)-z I)^{-1} f^{*}(s) d s d z \\
= & \frac{1}{2 i \pi} \int_{\gamma} \int_{0}^{t-\varepsilon} \frac{\sinh \sqrt{-z} s \cosh \sqrt{-z}(\delta-t)}{\sqrt{-z} \cosh \sqrt{-z} \delta} \\
& \times Q(t)(Q(t)-z I)^{-1}\left(f^{*}(s)-f^{*}(t)\right) d s d z \\
& +\frac{1}{2 i \pi} \int_{\gamma} \int_{t+\varepsilon}^{\delta} \frac{\sinh \sqrt{-z} t \cosh \sqrt{-z}(\delta-s)}{\sqrt{-z} \cosh \sqrt{-z} \delta} \\
& \times Q(t)(Q(t)-z I)^{-1}\left(f^{*}(s)-f^{*}(t)\right) d s d z \\
& -\frac{1}{2 i \pi} \int_{\gamma} \frac{\cosh \sqrt{-z}(t-\varepsilon) \cosh \sqrt{-z}(\delta-t)}{\cosh \sqrt{-z} \delta}(Q(t)-z I)^{-1} f^{*}(t) d z \\
& -\frac{1}{2 i \pi} \int_{\gamma} \frac{\sinh \sqrt{-z} t \sinh \sqrt{-z}(\delta-t-\varepsilon)}{\cosh \sqrt{-z} \delta}(Q(t)-z I)^{-1} f^{*}(t) d z \\
& +\frac{1}{2 i \pi} \int_{\gamma} \frac{\cosh \sqrt{-z}(\delta-t)}{\cosh \sqrt{-z} \delta}(Q(t)-z I)^{-1} f^{*}(t) d z .
\end{aligned}
$$

Thanks to (12), the first two integrals are absolutely convergent. The last one has been treated above. Observe that the convergence of the other integrals in $V_{\varepsilon}^{2}(t)$ and those of $V_{\varepsilon}^{3}(t)$ may be studied similarly. For example, the estimate

$$
\left|\frac{\sinh \sqrt{-z}(t-\varepsilon) \sinh \sqrt{-z}(\delta-t)}{\cosh \sqrt{-z} \delta}\right| \leq C e^{-\varepsilon|z|^{1 / 2}}
$$


leads to the convergence of the integral

$$
\frac{1}{2 i \pi} \int_{\gamma} \frac{\sinh \sqrt{-z}(t-\varepsilon) \sinh \sqrt{-z}(\delta-t)}{\cosh \sqrt{-z} \delta}(Q(t)-z I)^{-1} f^{*}(t-\varepsilon) d z
$$

for

$$
0<\varepsilon \leq t \leq \delta-\varepsilon<\delta
$$

Summing up, we obtain

$$
\begin{aligned}
v_{\varepsilon}^{\prime \prime}(t)+p(t) v^{\prime}(t)+ & Q(t) v(t) \\
& =\left(V_{\varepsilon}^{1}(t)+V^{4}(t)\right)+\left(V_{\varepsilon}^{2}(t)+Q(t) v(t)+V_{\varepsilon}^{3}(t)\right)+R_{\lambda}\left(f^{*}\right)(t) .
\end{aligned}
$$

Step 4. It is clear that $V_{\varepsilon}^{1}(t)+V^{4}(t)$ converges strongly to

$$
\begin{aligned}
P_{\lambda}\left(f^{*}\right)(t)= & -\frac{1}{2 i \pi} \int_{\gamma} \int_{0}^{\delta} K_{\sqrt{-z}}(t, s) \frac{\partial^{2}}{\partial t^{2}}(Q(t)-z I)^{-1} f^{*}(s) d s d z \\
& +\frac{1}{i \pi} \int_{\gamma} \int_{0}^{t} \frac{\sinh \sqrt{-z} s \sinh \sqrt{-z}(\delta-t)}{\cosh \sqrt{-z} \delta} \frac{\partial}{\partial t}(Q(t)-z I)^{-1} f^{*}(s) d s d z \\
& -\frac{1}{i \pi} \int_{\gamma} \int_{t}^{\delta} \frac{\cosh \sqrt{-z} t \cosh \sqrt{-z}(\delta-s)}{\cosh \sqrt{-z} \delta} \frac{\partial}{\partial t}(Q(t)-z I)^{-1} f^{*}(s) d s d z .
\end{aligned}
$$

Concerning the second term $V_{\varepsilon}^{2}(t)+Q(t) v(t)+V_{\varepsilon}^{3}(t)$, we have

$$
\lim _{\varepsilon \rightarrow 0}\left(V_{\varepsilon}^{2}(t)+Q(t) v(t)+V_{\varepsilon}^{3}(t)\right)=f^{*}(t)+\lim _{\varepsilon \rightarrow 0} W_{\varepsilon}^{2}(t),
$$

where

$$
\begin{aligned}
W_{\varepsilon}^{2}(t)= & -\frac{1}{2 i \pi} \int_{\gamma} \frac{\cosh \sqrt{z}(t-\varepsilon) \cosh \sqrt{-z}(\delta-t)}{\cosh \sqrt{-z} \delta}(Q(t)-z I)^{-1} f^{*}(t) d z \\
& -\frac{1}{2 i \pi} \int_{\gamma} \frac{\sinh \sqrt{-z} t \sinh \sqrt{-z}(\delta-t-\varepsilon)}{\cosh \sqrt{-z} \delta}(Q(t)-z I)^{-1} f^{*}(t) d z \\
& +\frac{1}{2 i \pi} \int_{\gamma} \frac{\sinh \sqrt{-z}(t-\varepsilon) \sinh \sqrt{-z}(\delta-t)}{\cosh \sqrt{-z} \delta}(Q(t)-z I)^{-1} f^{*}(t-\varepsilon) d z \\
& +\frac{1}{2 i \pi} \int_{\gamma} \frac{\cosh \sqrt{-z} t \cosh \sqrt{-z}(\delta-t-\varepsilon)}{\cosh \sqrt{-z} \delta}(Q(t)-z I)^{-1} f^{*}(t+\varepsilon) d z
\end{aligned}
$$

Step 5. Now, let us prove that $W_{\varepsilon}^{2}(t) \rightarrow 0$ as $\varepsilon \rightarrow 0$. For this purpose, observe 
that

$$
\begin{aligned}
& W_{\varepsilon}^{2}(t) \\
= & -\frac{1}{2 i \pi} \int_{\gamma} \frac{\cosh \sqrt{-z}(t-\varepsilon) \cosh \sqrt{-z}(\delta-t)}{\cosh \sqrt{-z} \delta}(Q(t)-z I)^{-1}\left(f^{*}(t)-f^{*}(t-\varepsilon)\right) d z \\
& -\frac{1}{2 i \pi} \int_{\gamma} \frac{\cosh \sqrt{-z}(2 t-\delta-\varepsilon)}{\cosh \sqrt{-z} \delta}(Q(t)-z I)^{-1} f^{*}(t-\varepsilon) d z \\
& -\frac{1}{2 i \pi} \int_{\gamma} \frac{\sinh \sqrt{-z} t \sinh \sqrt{-z}(\delta-t-\varepsilon)}{\cosh \sqrt{-z} \delta}(Q(t)-z I)^{-1}\left(f^{*}(t)-f^{*}(t+\varepsilon)\right) d z \\
& +\frac{1}{2 i \pi} \int_{\gamma} \frac{\cosh \sqrt{-z}(2 t-\delta+\varepsilon)}{\cosh \sqrt{-z} \delta}(Q(t)-z I)^{-1} f^{*}(t+\varepsilon) d z \\
= & -\frac{1}{2 i \pi} \int_{\gamma} \frac{\cosh \sqrt{-z}(t-\varepsilon) \cosh \sqrt{-z}(\delta-t)}{\cosh \sqrt{-z} \delta}(Q(t)-z I)^{-1}\left(f^{*}(t)-f^{*}(t-\varepsilon)\right) d z \\
& -\frac{1}{2 i \pi} \int_{\gamma} \frac{\cosh \sqrt{-z}(2 t-\delta-\varepsilon)}{\cosh \sqrt{-z} \delta}(Q(t)-z I)^{-1}\left(f^{*}(t-\varepsilon)-f^{*}(t+\varepsilon)\right) d z \\
& -\frac{1}{2 i \pi} \int_{\gamma} \frac{\sinh \sqrt{-z} t \sinh \sqrt{-z}(\delta-t-\varepsilon)}{\cosh \sqrt{-z} \delta}(Q(t)-z I)^{-1}\left(f^{*}(t)-f^{*}(t+\varepsilon)\right) d z \\
& +\frac{1}{2 i \pi} \int_{\gamma} \frac{\cosh \sqrt{-z}(2 t-\delta+\varepsilon)-\cosh \sqrt{-z}(2 t-\delta-\varepsilon)}{\cosh \sqrt{-z} \delta}(Q(t)-z I)^{-1} f^{*}(t+\varepsilon) d z .
\end{aligned}
$$

Regarding the first term, we have

$$
\begin{gathered}
\left\|\frac{1}{2 i \pi} \int_{\gamma} \frac{\cosh \sqrt{-z}(t-\varepsilon) \cosh \sqrt{-z}(\delta-t)}{\cosh \sqrt{-z} \delta}(Q(t)-z I)^{-1}\left(f^{*}(t)-f^{*}(t-\varepsilon)\right) d z\right\| \\
\leq C \varepsilon^{\beta}\left(\int_{z \in \gamma,|z| \geq 1 / \varepsilon^{2}} e^{-\varepsilon|z|^{1 / 2}} \frac{|d z|}{|z|}+\int_{z \in \gamma,|z| \leq 1 / \varepsilon^{2}} \frac{|d z|}{|z|}\right)\|f\|_{C^{\beta}(E)} \\
\leq C \varepsilon^{\beta}\left(\int_{1}^{\infty} e^{-\sigma} \frac{2 \sigma d \sigma}{\sigma^{2}}+\int_{r_{0}}^{1 / \varepsilon^{2}} \frac{d \rho}{\rho}\right)\|f\|_{C^{\beta}(E)}
\end{gathered}
$$

where $r_{0}$ has been introduced in (3). We deduce the convergence of this integral to 0 when $\varepsilon \rightarrow 0$. It is easy to see that we have the same result for the second integral 
since

$$
\begin{gathered}
\left\|\frac{1}{2 i \pi} \int_{\gamma} \frac{\cosh \sqrt{-z}(2 t-\delta-\varepsilon)}{\cosh \sqrt{-z} \delta}(Q(t)-z I)^{-1}\left(f^{*}(t-\varepsilon)-f^{*}(t+\varepsilon)\right) d z\right\| \\
\leq C \varepsilon^{\beta}\left(\int_{\gamma} \frac{e^{-|z|^{1 / 2}(2 \delta-2 t+\varepsilon)}+e^{-|z|^{1 / 2}(2 t-\varepsilon)}|d z|}{|z|}\right)\|f\|_{C^{\beta}(E)} \\
\leq C \varepsilon^{\beta}\left(\int_{\gamma} \frac{e^{-|z|^{1 / 2}(2 \delta-2 t)}+e^{-|z|^{1 / 2}(2 t)}|d z|}{|z|}\right)\|f\|_{C^{\beta}(E)},
\end{gathered}
$$

with $0<\varepsilon \leq t \leq \delta-\varepsilon<\delta$. The third integral can be treated as the first one. Finally, about the fourth integral observe that

$$
\begin{aligned}
& \frac{\cosh \sqrt{-z}(2 t-\delta+\varepsilon)-\cosh \sqrt{-z}(2 t-\delta-\varepsilon)}{\cosh \sqrt{-z} \delta} \\
& =\frac{e^{-\sqrt{-z}(2 \delta-2 t-\varepsilon)}-e^{-\sqrt{-z}(2 \delta-2 t+\varepsilon)}}{1+e^{-2 \delta \sqrt{-z}}}+\frac{e^{-\sqrt{-z}(2 t+\varepsilon)}-e^{-\sqrt{-z}(2 t-\varepsilon)}}{1+e^{-2 \delta \sqrt{-z}}},
\end{aligned}
$$

and

$$
\begin{array}{r}
\left\|\frac{1}{2 i \pi} \int_{\gamma} \frac{e^{-\sqrt{-z}(2 \delta-2 t-\varepsilon)}-e^{-\sqrt{-z}(2 \delta-2 t+\varepsilon)}}{1+e^{-2 \delta \sqrt{-z}}}(Q(t)-z I)^{-1} f^{*}(t+\varepsilon) d z\right\| \\
\leq C \int_{\gamma} e^{-|z|^{1 / 2}(2 \delta-2 t-\varepsilon)}\left(1-e^{-2 \varepsilon|z|^{1 / 2}}\right) \frac{|d z|}{|z|}\left\|f^{*}\right\|_{C^{\beta}(E)} .
\end{array}
$$

The latter tends to 0 by Lebesgue dominated convergence theorem since

$$
e^{-|z|^{1 / 2}(2 \delta-2 t-\varepsilon)} \frac{\left(1-e^{-2 \varepsilon|z|^{1 / 2}}\right)}{|z|} \leq \frac{2 e^{-|z|^{1 / 2}(2 \delta-2 t)}}{|z|}
$$

for all $0<\varepsilon \leq t \leq \delta-\varepsilon<\delta$. By the same way, we treat the last term.

Step 6. Summing up, we obtain the strong convergence

$$
v_{\varepsilon}^{\prime}(t) \rightarrow v^{\prime}(t) \quad \text { and } \quad v_{\varepsilon}^{\prime \prime}(t) \rightarrow-Q(t) v(t)+P_{\lambda}\left(f^{*}\right)(t)+f^{*}(t)
$$

as $\varepsilon \rightarrow 0$. Hence

$$
v^{\prime \prime}(t)=-Q(t) v(t)+P_{\lambda}\left(f^{*}\right)(t)+f^{*}(t),
$$

or

$$
\begin{aligned}
v^{\prime \prime}(t)+p(t) v^{\prime}(t)+Q(t) v(t) & =P_{\lambda}\left(f^{*}\right)(t)+p(t) v^{\prime}(t)+f^{*}(t) \\
& =P_{\lambda}\left(f^{*}\right)(t)+R_{\lambda}\left(f^{*}\right)(t)+f^{*}(t) .
\end{aligned}
$$


On the other hand, we have

$$
\begin{aligned}
& \left\|\int_{\gamma} \int_{0}^{\delta} K_{\sqrt{-z}}(t, s) \frac{\partial^{2}}{\partial t^{2}}(Q(t)-z I)^{-1} f^{*}(s) d s d z\right\| \\
& \leq C \int_{\gamma} \frac{1}{|z||z+\lambda|^{\rho}}|d z|\left\|f^{*}\right\|_{C(E)} \leq\left(C / \lambda^{\rho}\right)\left\|f^{*}\right\|_{C(E)}, \\
& \left\|\frac{1}{2 i \pi} \int_{\gamma} \int_{0}^{\delta} \frac{\partial}{\partial t} K_{\sqrt{-z}}(t, s) \frac{\partial}{\partial t}(Q(t)-z I)^{-1} f^{*}(s) d s d z\right\| \\
& \leq C \int_{\gamma} \frac{1}{|z|^{1 / 2}|z+\lambda|^{1 / 2+\alpha}}|d z|\left\|f^{*}\right\|_{C(E)} \leq\left(C / \lambda^{\alpha}\right)\left\|f^{*}\right\|_{C(E)}, \\
& \left\|-\frac{1}{2 i \pi} \int_{\gamma} \int_{0}^{\delta} K_{\sqrt{-z}}(t, s) p(t) \frac{\partial}{\partial t}(Q(t)-z I)^{-1} f^{*}(s) d s d z\right\| \\
& \leq C \int_{\gamma} \frac{1}{|z|} \frac{1}{|z+\lambda|^{\alpha+1 / 2}} d|z|\left\|f^{*}\right\|_{C(E)} \leq\left(C / \lambda^{\alpha+1 / 2}\right)\left\|f^{*}\right\|_{C(E)},
\end{aligned}
$$

and

$$
\begin{aligned}
\|-\frac{1}{2 i \pi} \int_{\gamma} \int_{0}^{\delta} \frac{\partial}{\partial t} & K_{\sqrt{-z}}(t, s) p(t)(Q(t)-z I)^{-1} f^{*}(s) d s d z \| \\
& \leq C \int_{\gamma} \frac{d|z|}{|z|^{1 / 2}|z+\lambda|}\left\|f^{*}\right\|_{C(E)} \leq\left(C / \lambda^{1 / 2}\right)\left\|f^{*}\right\|_{C(E)} .
\end{aligned}
$$

Therefore

$$
\left\|\left(P_{\lambda}+R_{\lambda}\right)\right\|_{L(C([0, \delta] ; E))} \leq C\left(1 / \lambda^{\alpha+1 / 2}+1 / \lambda^{\rho}+1 / \lambda^{1 / 2}\right)
$$

and the assertion is proved.

Proposition 1.5. Suppose that $\varphi^{*} \in D(Q(0))$. Then, under the assumptions (6)-(9), the function $d(\cdot, Q(\cdot)) \varphi^{*}$ belongs to $\left.\left.C^{2}(] 0, \delta\right] ; E\right)$.

Proof. Recall that, for $t \in] 0, \delta]$

$$
d(t, Q(t)) \varphi^{*}=\frac{1}{2 i \pi} \int_{\gamma} c_{\sqrt{-z}}(t)(Q(t)-z I)^{-1} \varphi^{*} d z
$$

and if $\varphi^{*} \in D(Q(0))$

$$
d(t, Q(t)) \varphi^{*} \in D(Q(t)) .
$$


On the other hand

$$
\begin{aligned}
d^{\prime}(t, Q(t)) \varphi^{*}= & \frac{1}{2 i \pi} \int_{\gamma} c_{\sqrt{-z}}(t) \frac{\partial}{\partial t}(Q(t)-z I)^{-1} \varphi^{*} d z \\
& -\frac{1}{2 i \pi} \int_{\gamma} \sqrt{-z} s \sqrt{-z}(\delta-t)(Q(t)-z I)^{-1} \varphi^{*} d z
\end{aligned}
$$

and

$$
\begin{aligned}
d^{\prime \prime}(t, Q(t)) \varphi^{*}= & \frac{1}{2 i \pi} \int_{\gamma} c_{\sqrt{-z}}(t) \frac{\partial^{2}}{\partial t^{2}}(Q(t)-z I)^{-1} \varphi^{*} d z \\
& -\frac{1}{i \pi} \int_{\gamma} \sqrt{-z} s \sqrt{-z}(\delta-t) \frac{\partial}{\partial t}(Q(t)-z I)^{-1} \varphi^{*} d z \\
& -\frac{1}{2 i \pi} \int_{\gamma} c_{\sqrt{-z}}(t) z(Q(t)-z I)^{-1} \varphi^{*} d z \\
= & \frac{1}{2 i \pi} \int_{\gamma} c_{\sqrt{-z}}(t) \frac{\partial^{2}}{\partial t^{2}}(Q(t)-z I)^{-1} \varphi^{*} d z \\
& -\frac{1}{i \pi} \int_{\gamma} \sqrt{-z} s \sqrt{-z}(\delta-t) \frac{\partial}{\partial t}(Q(t)-z I)^{-1} \varphi^{*} d z \\
& -\frac{1}{2 i \pi} \int_{\gamma} c_{\sqrt{-z}}(t) Q(t)(Q(t)-z I)^{-1} \varphi^{*} d z
\end{aligned}
$$

We obtain the desired regularity for $t \in] 0, \delta]$.

In the sequel, we will put

$$
\begin{aligned}
d^{\prime \prime}(t, Q(t)) \varphi^{*}+Q(t) d(t, Q(t)) \varphi^{*}= & \frac{1}{2 i \pi} \int_{\gamma} c_{\sqrt{-z}}(t) \frac{\partial^{2}}{\partial t^{2}}(Q(t)-z I)^{-1} \varphi^{*} d z \\
& -\frac{1}{i \pi} \int_{\gamma} \sqrt{-z} s \sqrt{-z}(\delta-t) \frac{\partial}{\partial t}(Q(t)-z I)^{-1} \varphi^{*} d z \\
:= & F_{\lambda}\left(\varphi^{*}\right)(t),
\end{aligned}
$$

and

$$
\begin{aligned}
p(t) d^{\prime}(t, Q(t)) \varphi^{*}= & \frac{1}{2 i \pi} \int_{\gamma} c_{\sqrt{-z}}(t) p(t) \frac{\partial}{\partial t}(Q(t)-z I)^{-1} \varphi^{*} d z \\
& -\frac{1}{2 i \pi} \int_{\gamma} \sqrt{-z} s_{\sqrt{-z}}(\delta-t) p(t)(Q(t)-z I)^{-1} \varphi^{*} d z \\
:= & G_{\lambda}\left(\varphi^{*}\right)(t)
\end{aligned}
$$

thus

$$
d^{\prime \prime}(t, Q(t)) \varphi^{*}+p(t) d^{\prime}(t, Q(t)) \varphi^{*}+Q(t) d(t, Q(t)) \varphi^{*}=F_{\lambda}\left(\varphi^{*}\right)(t)+G_{\lambda}\left(\varphi^{*}\right)(t) .
$$


Remark 1.6. The regularity up to 0 for $d^{\prime \prime}(\cdot, Q(\cdot)) \varphi^{*}$ and $Q(\cdot) d(\cdot, Q(\cdot)) \varphi^{*}$ will be treated below by associating $d$ and $v$. This is not trivial and will need a compatibility condition.

Now, let us study the integral

$$
n(t, Q(t)) \psi^{*}=\frac{1}{2 i \pi} \int_{\gamma} s_{\sqrt{-z}}(t) \frac{(Q(t)-z I)^{-1}}{\sqrt{-z}} \psi^{*} d z .
$$

Since

$$
\left\|s_{\sqrt{-z}}(t) \frac{(Q(t)-z I)^{-1}}{\sqrt{-z}} \psi^{*}\right\|_{E}=O\left(\frac{e^{-(\delta-t)|z|^{1 / 2} \sin \left(\theta_{0} / 2\right)}}{|z|^{1+1 / 2}}\right)\left\|\psi^{*}\right\|_{E},
$$

for each $t \geq 0$, the vector function $n(t, Q(t)) \psi^{*}$ is well defined and it is continuous on $[0, \delta]$ for each $\psi^{*} \in E$. Furthermore, this function is regular for $t \in[0, \delta[$. In order to have more regularity on the closed interval we assume that

$$
\exists \epsilon>0: D_{q(\delta)}(1 / 2+\epsilon,+\infty)=D_{q(0)}(1 / 2+\epsilon,+\infty) .
$$

Observe that our differentiability hypotheses on the resolvents show that this condition is natural when $\delta \rightarrow 0$.

Proposition 1.7. Under the assumptions (6)-(9) and the condition

$$
\psi^{*} \in D_{q(\delta)}(1 / 2+\epsilon,+\infty)=D_{q(0)}(1 / 2+\epsilon,+\infty)
$$

the function $n(\cdot, Q(\cdot)) \psi^{*}$ belongs to $C^{2}([0, \delta] ; E)$.

Proof. First, note that it is not difficult to prove that $n(\cdot, Q(\cdot)) \psi^{*} \in C^{2}([0, \delta] ; E)$ if and only if $Q(\cdot) n(\cdot, Q(\cdot)) \psi^{*}$ is continuous. The following relation holds true for $t \in\left[0, \delta\left[\right.\right.$ and $\psi^{*} \in E$ :

$$
Q(t) n(t, Q(t)) \psi^{*}=\frac{1}{2 i \pi} \int_{\gamma} s_{\sqrt{-z}}(t) \frac{Q(t)(Q(t)-z I)^{-1}}{\sqrt{-z}} \psi^{*} d z .
$$

Moreover, the integral is absolutely convergent. We have to study the case when $t=\delta$. Let us consider $t \in[0, \delta[$. Then

$$
\begin{aligned}
& Q(t) n(t, Q(t)) \psi^{*} \\
& \begin{aligned}
=\frac{1}{2 i \pi} \int_{\gamma} \frac{s \sqrt{-z}(t)}{\sqrt{-z}}\left(Q(t)(Q(t)-z I)^{-1}-Q(\delta)(Q(\delta)-z I)^{-1}\right) \psi^{*} d z \\
+\frac{1}{2 i \pi} \int_{\gamma} \frac{s \sqrt{-z}(t)}{\sqrt{-z}} Q(\delta)(Q(\delta)-z I)^{-1} \psi^{*} d z .
\end{aligned}
\end{aligned}
$$

From

$$
Q(t)(Q(t)-z I)^{-1}-Q(\delta)(Q(\delta)-z I)^{-1}=z\left((Q(t)-z I)^{-1}-(Q(\delta)-z I)^{-1}\right)
$$


it follows that

$$
\begin{aligned}
Q(t) n(t, Q(t)) \psi^{*}= & \frac{1}{2 i \pi} \int_{\gamma} \sqrt{-z} s_{\sqrt{-z}}(t) \int_{t}^{\delta} \frac{\partial}{\partial r}(Q(r)-z I)^{-1} \psi^{*} d r d z \\
& +\frac{1}{2 i \pi} \int_{\gamma} \frac{s_{\sqrt{-z}}(t)}{\sqrt{-z}} Q(\delta)(Q(\delta)-z I)^{-1} \psi^{*} d z \\
:= & J_{1}+J_{2} .
\end{aligned}
$$

Let $J_{1}=J_{1}^{+}+J_{1}^{-}$where $J_{1}^{+}$and $J_{1}^{-}$denote respectively the integrals on the parts $\gamma_{+}=\left\{z \in \gamma:|z| \geq 1 /(\delta-t)^{2}\right\}$ and $\gamma_{-}=\left\{z \in \gamma:|z| \leq 1 /(\delta-t)^{2}\right\}$ of $\gamma$.

We write

$$
J_{1}^{+}:=J_{11}^{+}+J_{12}^{+}+J_{13}^{+},
$$

where

$$
\begin{aligned}
J_{11}^{+}= & \frac{1}{2 i \pi} \int_{\gamma_{+}} \sqrt{-z} s_{\sqrt{-z}}(t) \int_{t}^{\delta}\left(\frac{\partial}{\partial r}(Q(r)-z I)^{-1}-\frac{\partial}{\partial t}(Q(t)-z I)^{-1}\right) \psi^{*} d r d z \\
J_{12}^{+}= & \frac{1}{2 i \pi} \int_{\gamma_{+}}(\delta-t) \sqrt{-z} s_{\sqrt{-z}}(t)\left(\frac{\partial}{\partial t}(Q(t)-z I)^{-1}-\frac{\partial}{\partial t}(Q(t)-z I)_{\mid t=\delta}^{-1}\right) \psi^{*} d z \\
J_{13}^{+}= & \frac{1}{2 i \pi} \int_{\gamma_{+}}(\delta-t) \sqrt{-z} s_{\sqrt{-z}}(t) Q(\delta)(Q(\delta)-z I)^{-1} \\
& \times \frac{d}{d t} Q(t)_{\mid t=\delta}^{-1} Q(\delta)(Q(\delta)-z I)^{-1} \psi^{*} d z .
\end{aligned}
$$

Then (in virtue of subsection 0.2 and remark 1.3)

$$
\begin{aligned}
\left\|J_{11}^{+}\right\| \leq & C \int_{\gamma_{+}}|z|^{1 / 2} e^{-|z|^{1 / 2}(\delta-t)} \int_{t}^{\delta} \frac{(r-t)^{2 \eta}}{|z|^{\rho+1 / 2}} d r|d z|\left\|\psi^{*}\right\|_{E} \\
& +C \int_{\gamma_{+}}|z|^{1 / 2} e^{-|z|^{1 / 2}(\delta-t)} \int_{t}^{\delta} \frac{(r-t)}{|z|^{2 \alpha}} d r|d z|\left\|\psi^{*}\right\|_{E} \\
\leq & C \int_{1}^{\infty}(\delta-t)^{2 \eta} \frac{\sigma^{2} e^{-\sigma}}{\left(\frac{\sigma^{2}}{(\delta-t)^{2}}\right)^{\rho+1 / 2}} \frac{d \sigma}{(\delta-t)^{2}}\left\|\psi^{*}\right\|_{E} \\
& +C \int_{1}^{\infty} \frac{\sigma^{2} e^{-\sigma}}{(\delta-t)} \frac{1}{\left(\frac{\sigma^{2}}{(\delta-t)^{2}}\right)^{2 \alpha}} d \sigma\left\|\psi^{*}\right\|_{E} \\
\leq & C\left((\delta-t)^{2 \eta+2 \rho-1}+(\delta-t)^{4 \alpha-1}\right)\left\|\psi^{*}\right\|_{E}
\end{aligned}
$$

and $2 \eta+2 \rho-1>0,4 \alpha-1>0$. A similar estimate can be shown for $J_{12}^{+}$. Concerning the third integral $J_{13}^{+}$, we obtain, thanks to the regularity of $\psi^{*}$

$$
\left\|J_{13}^{+}\right\| \leq C(\delta-t)^{2 \alpha+2 \epsilon}\left\|\psi^{*}\right\|_{D_{q(0)}(1 / 2+\epsilon,+\infty)} .
$$


The same calculations lead to a similar estimate for $\left\|J_{1}^{-}\right\|$.

For $J_{2}$, we have

$$
\begin{aligned}
\left\|J_{2}\right\| & \leq C \int_{\gamma} \frac{1}{|z|^{1 / 2}} \frac{1}{|z|^{1 / 2+\epsilon}}|d z|\left\|\psi^{*}\right\|_{D_{q(0)}(1 / 2+\epsilon,+\infty)} \\
& \leq C\left\|\psi^{*}\right\|_{D_{q(0)}(1 / 2+\epsilon,+\infty)} .
\end{aligned}
$$

Summing up, the previous estimates justify the continuity of $Q(\cdot) n(\cdot, Q(\cdot)) \psi^{*}$ at the point $\delta$. This establishes the proposition.

Remark 1.8. (i) The term $J_{13}^{+}$is regular for $\psi^{*} \in D\left((-Q(\delta))^{1 / 2}\right)$ since, it is possible to write it as

$$
\begin{aligned}
J_{13}^{+}= & \frac{1}{2 i \pi} \int_{\gamma_{+}}(\delta-t) \sqrt{-z} s_{\sqrt{-z}}(t) \\
& \times\left[Q(\delta)(Q(\delta)-z I)^{-1} \frac{d}{d t} Q(t)_{\mid t=\delta}^{-1}(-Q(\delta))^{1 / 2}(Q(\delta)-z I)^{-1}\right. \\
& \left.\times(-Q(\delta))^{1 / 2} \psi^{*}\right] d z
\end{aligned}
$$

and then

$$
\begin{aligned}
\left\|J_{13}^{+}\right\| & \leq C \int_{\gamma_{+}}(\delta-t)|z|^{1 / 2} e^{-|z|^{1 / 2}(\delta-t)} \frac{1}{|z|^{\alpha+1 / 2}} \frac{1}{|z|^{1 / 2}}|d z|\left\|\psi^{*}\right\|_{D\left((-Q(\delta))^{1 / 2}\right)} \\
& \leq C(\delta-t)^{2 \alpha}\left\|\psi^{*}\right\|_{D\left((-Q(\delta))^{1 / 2}\right)} .
\end{aligned}
$$

(ii) The same argument holds for

$$
J_{2}=-\frac{1}{2 i \pi} \int_{\gamma} \frac{s_{\sqrt{-z}}(t)}{\sqrt{-z}}(Q(\delta)-z I)^{-1} \psi^{*} d z .
$$

As in the proof of Lemma 1.2, statement (iv), we have

$$
\begin{aligned}
J_{2}=-\left(I+e^{-2 \delta(-Q(0))^{1 / 2}}\right)^{-1} & \\
& \times\left\{\left(I-e^{\left.-2(-Q(0))^{1 / 2} t\right)}\right) e^{-(-Q(0))^{1 / 2}(\delta-t)}(-Q(\delta))^{1 / 2} \psi^{*}\right\}
\end{aligned}
$$

and applying Sinestrari [11] again, we obtain the continuity of $J_{2}$ if and only if

$$
(-Q(\delta))^{1 / 2} \psi^{*} \in \overline{D\left((-Q(\delta))^{1 / 2}\right)}=\overline{D(Q(\delta))} .
$$

But in this work, we will work with the following sufficient hypothesis

$$
\psi^{*} \in D_{q(\delta)}(1 / 2+\epsilon,+\infty)=D_{q(0)}(1 / 2+\epsilon,+\infty)
$$

which is more easy to handle. 
The following term is handled similarly

$$
\begin{aligned}
n^{\prime}(t, Q(t)) \psi^{*}=\frac{1}{2 i \pi} \int_{\gamma} \frac{s \sqrt{-z}(t)}{\sqrt{-z}} \frac{\partial}{\partial t}(Q(t) & -z I)^{-1} \psi^{*} d z \\
& +\frac{1}{2 i \pi} \int_{\gamma} c_{\sqrt{-z}}(\delta-t)(Q(t)-z I)^{-1} \psi^{*} d z .
\end{aligned}
$$

We will set

$$
p(t) n^{\prime}(t, Q(t)) \psi^{*}=T_{\lambda}\left(\psi^{*}\right)(t)
$$

and

$$
\begin{aligned}
n^{\prime \prime}(t, Q(t)) \psi^{*}+Q(t) n(t, Q(t)) \psi^{*}= & \frac{1}{2 i \pi} \int_{\gamma} \frac{s \sqrt{-z}(t)}{\sqrt{-z}} \frac{\partial^{2}}{\partial t^{2}}(Q(t)-z I)^{-1} \psi^{*} d z \\
& +\frac{2}{2 i \pi} \int_{\gamma} c_{\sqrt{-z}}(\delta-t) \frac{\partial}{\partial t}(Q(t)-z I)^{-1} \psi^{*} d z \\
:= & S_{\lambda}\left(\psi^{*}\right)(t) .
\end{aligned}
$$

The following lemma is needed in order to study the optimal regularity of the solution we are looking for.

Lemma 1.9. Let $\varphi^{*} \in D_{q(0)}(1 / 2,+\infty)$. Under the assumptions $(6)-(9)$, we have

$$
\frac{1}{2 i \pi} \int_{\gamma} c_{\sqrt{-z}}(t) \frac{\partial}{\partial t}(Q(t)-z I)^{-1} \varphi^{*} d z \rightarrow \frac{1}{2 i \pi} \int_{\gamma} \frac{\partial}{\partial t}(Q(t)-z I)_{\mid t=0}^{-1} \varphi^{*} d z=0
$$

as $t \rightarrow 0$. (This result is then valid for $\varphi^{*} \in D(Q(0))=D(q(0))$.)

Proof. It is easy to see that

$$
\begin{aligned}
\frac{1}{2 i \pi} \int_{\gamma} c_{\sqrt{-z}}(t) \frac{\partial}{\partial t}(Q(t)-z I)^{-1} \varphi^{*} d z \\
=\frac{1}{2 i \pi} \int_{\gamma} c_{\sqrt{-z}}(t)\left(\frac{\partial}{\partial t}(Q(t)-z I)^{-1}-\frac{\partial}{\partial t}(Q(t)-z I)_{\mid t=0}^{-1}\right) \varphi^{*} d z \\
\quad+\frac{1}{2 i \pi} \int_{\gamma} c_{\sqrt{-z}}(t) Q(0)(Q(0)-z I)^{-1} \frac{d}{d t} Q(t)_{\mid t=0}^{-1} Q(0)(Q(0)-z I)^{-1} \varphi^{*} d z \\
:=I_{1}(t)+I_{2}(t) .
\end{aligned}
$$

By the dominated convergence theorem together with

$$
\begin{aligned}
\| Q(0)(Q(0)-z I)^{-1} \frac{d}{d t} Q(t)_{\mid t=0}^{-1} Q(0)(Q(0) & -z I)^{-1} \varphi^{*} \| \\
& =O\left(\frac{1}{|z|^{\alpha+1 / 2+1 / 2}}\right)\left\|\varphi^{*}\right\|_{D_{q(0)}(1 / 2,+\infty)}
\end{aligned}
$$


and integrating on the right-hand side of $\gamma$, we prove that $I_{2}(t) \rightarrow 0$ as $t \rightarrow 0$.

Put $I_{1}=I_{1}^{+}+I_{1}^{-}$where $I_{1}^{+}(t)$ and $I_{1}^{-}(t)$ denote respectively the integrals on the parts $\gamma_{+}=\left\{z \in \gamma:|z| \geq 1 / t^{2}\right\}$ and $\gamma_{-}=\left\{z \in \gamma:|z| \leq 1 / t^{2}\right\}$ of $\gamma$. It follows that

$$
\begin{aligned}
\left\|I_{1}^{+}(t)\right\| & =\left\|\frac{1}{2 i \pi} \int_{\gamma_{+}} c_{\sqrt{-z}}(t)\left(\frac{\partial}{\partial t}(Q(t)-z I)^{-1}-\frac{\partial}{\partial t}(Q(t)-z I)_{\mid t=0}^{-1}\right) \varphi^{*} d z\right\| \\
& \leq C \int_{\gamma_{+}} e^{-|z|^{1 / 2} t} \frac{t^{2 \eta}}{|z|^{\rho+1 / 2}}|d z|\left\|\varphi^{*}\right\|_{E}+C \int_{\gamma_{+}} e^{-|z|^{1 / 2} t} \frac{t}{|z|^{2 \alpha}}|d z|\left\|\varphi^{*}\right\|_{E} \\
& \leq C\left(t^{2 \eta+2 \rho-1}+t^{4 \alpha-1}\right)\left\|\varphi^{*}\right\|_{E} .
\end{aligned}
$$

Similarly, for $I_{1}^{-}$, we have

$$
\begin{aligned}
I_{1}^{-}(t)= & \frac{1}{2 i \pi} \int_{\gamma-} c_{\sqrt{-z}}(t)\left(\frac{\partial}{\partial t}(Q(t)-z I)^{-1}-\frac{\partial}{\partial t}(Q(t)-z I)_{\mid t=0}^{-1}\right) \varphi^{*} d z \\
= & \frac{1}{2 i \pi} \int_{\gamma-}\left(c_{\sqrt{-z}}(t)-1\right)\left(\frac{\partial}{\partial t}(Q(t)-z I)^{-1}-\frac{\partial}{\partial t}(Q(t)-z I)_{\mid t=0}^{-1}\right) \varphi^{*} d z \\
& +\frac{1}{2 i \pi} \int_{\gamma-}\left(\frac{\partial}{\partial t}(Q(t)-z I)^{-1}-\frac{\partial}{\partial t}(Q(t)-z I)_{\mid t=0}^{-1}\right) \varphi^{*} d z, \\
I_{1}^{-}(t)= & m_{1}(t)+m_{2}(t) .
\end{aligned}
$$

The same estimate for $m_{2}$ is obtained as above, after closing the curve $\gamma^{-}$on the right-hand side. Regarding $m_{1}$, we have

$$
\begin{aligned}
\left\|m_{1}(t)\right\|= & \left\|\frac{1}{2 i \pi} \int_{\gamma-}\left(c_{\sqrt{-z}}(t)-1\right)\left(\frac{\partial}{\partial t}(Q(t)-z I)^{-1}-\frac{\partial}{\partial t}(Q(t)-z I)_{\mid t=0}^{-1}\right) \varphi^{*} d z\right\| \\
= & \| \frac{1}{2 i \pi} \int_{\gamma-} \int_{0}^{t} \sqrt{-z} s_{\sqrt{-z}}(t-r) \\
& \times\left(\frac{\partial}{\partial t}(Q(t)-z I)^{-1}-\frac{\partial}{\partial t}(Q(t)-z I)^{-1}\right) \varphi^{*} d r d z \| \\
\left\|m_{1}(t)\right\| \leq & C \int_{r_{0}}^{1 / t^{2}} t|z|^{1 / 2} \frac{t^{2 \eta}}{|z|^{\rho+1 / 2}} d|z|\left\|\varphi^{*}\right\|_{E} \leq C t^{2 \eta+2 \rho-1}\left\|\varphi^{*}\right\|_{E} .
\end{aligned}
$$

This ends the proof of the lemma.

\subsection{The system verified by the solution}

We have seen that under the hypotheses upon the resolvents of $Q(t), \varphi^{*}$ and $\psi^{*}$, if $f^{*}$ is Hölderian then the function given by (11) is in $C(] 0, \delta\left[; D(Q(\cdot)) \cap C^{2}(] 0, \delta[; E)\right.$. 
Suppose that $u$ is a solution of the complete abstract problem, that is,

$$
\begin{aligned}
u^{\prime \prime}(t)+p(t) u^{\prime}(t)+Q(t) u(t)= & f(t)=v^{\prime \prime}(t)+p(t) v^{\prime}(t)+Q(t) v(t) \\
& +d^{\prime \prime}(t, Q(t)) \varphi^{*}+p(t) d^{\prime}(t, Q(t)) \varphi^{*}+Q(t) d(t, Q(t)) \varphi^{*} \\
& +n^{\prime \prime}(t, Q(t)) \psi^{*}+p(t) n^{\prime}(t, Q(t)) \psi^{*}+Q(t) n(t, Q(t)) \psi^{*} .
\end{aligned}
$$

Then

$\left.f^{*}(t)+\left(P_{\lambda}+R_{\lambda}\right)\left(f^{*}\right)(t)+\left(F_{\lambda}+G_{\lambda}\right)\left(\varphi^{*}\right)(t)+\left(S_{\lambda}+T_{\lambda}\right)\left(\psi^{*}\right)(t)=f(t), \quad t \in\right] 0, \delta[$,

where

$$
\left\{\begin{array}{l}
F_{\lambda}\left(\varphi^{*}\right)(t):=d^{\prime \prime}(t, Q(t)) \varphi^{*}+Q(t) d(t, Q(t)) \varphi^{*}, \\
G_{\lambda}\left(\varphi^{*}\right)(t):=p(t) d^{\prime}(t, Q(t)) \varphi^{*}, \\
S_{\lambda}\left(\psi^{*}\right)(t):=n^{\prime \prime}(t, Q(t)) \psi^{*}+Q(t) n(t, Q(t)) \psi^{*}, \\
T_{\lambda}\left(\psi^{*}\right)(t):=p(t) n^{\prime}(t, Q(t)) \psi^{*} .
\end{array}\right.
$$

We have to express that the solution satisfies $u(0)=\varphi$ and $u^{\prime}(\delta)=\psi$.

It is easy to see that

$$
v(0)=0, \quad n(0)=0
$$

and, for $\varphi^{*} \in D(Q(0))=D(q(0))$,

$$
d(0)=\frac{1}{2 i \pi} \int_{\gamma} \frac{(Q(0)-z I)^{-1} Q(0) \varphi^{*}}{z} d z=\varphi^{*} .
$$

In view of the expressions of $v^{\prime}(t), d^{\prime}(t)$, and $n^{\prime}(t)$, using the relation

$$
\frac{1}{2 i \pi} \int_{\gamma} c_{\sqrt{-z}}(\delta-\tau)(Q(\delta)-z I)^{-1} \psi^{*} d z \rightarrow \psi^{*} \quad \text { as } \tau \rightarrow \delta
$$

(since $\psi^{*} \in D_{q(\delta)}(1 / 2+\epsilon,+\infty)=D_{q(0)}(1 / 2+\epsilon,+\infty)$, see lemma 1.2), we deduce that

$$
\begin{aligned}
& v^{\prime}(\delta)=-\left.\frac{1}{2 i \pi} \int_{\gamma} \int_{0}^{\delta} \frac{s \sqrt{-z}(\xi)}{\sqrt{-z}} \frac{\partial}{\partial t}(Q(t)-z I)^{-1}\right|_{t=\delta} f^{*}(\xi) d \xi d z:=V_{\lambda}\left(f^{*}\right), \\
& d^{\prime}(\delta)=\left.\frac{1}{2 i \pi} \int_{\gamma} \frac{1}{\cosh \sqrt{-z} \delta} \frac{\partial}{\partial t}(Q(t)-z I)^{-1}\right|_{t=\delta} \varphi^{*} d z:=D_{\lambda}\left(\varphi^{*}\right),
\end{aligned}
$$

and

$$
\begin{aligned}
n^{\prime}(\delta) & =\left.\frac{1}{2 i \pi} \int_{\gamma} \frac{s \sqrt{-z}(\delta)}{\sqrt{-z}} \frac{\partial}{\partial t}(Q(t)-z I)^{-1}\right|_{t=\delta} \psi^{*} d z+\psi^{*} \\
& :=\psi^{*}+N_{\lambda}\left(\psi^{*}\right) .
\end{aligned}
$$

So, the function

$$
u(t)=d\left(t, Q((t)) \varphi^{*}+n\left(t, Q((t)) \psi^{*}+v\left(t, Q((t)) f^{*}\right.\right.\right.
$$


satisfies the system

$$
\left\{\begin{array}{l}
f^{*}(t)+P_{\lambda}\left(f^{*}\right)(t)+R_{\lambda}\left(f^{*}\right)(t)+F_{\lambda}\left(\varphi^{*}\right)(t) \\
\left.\quad+G_{\lambda}\left(\varphi^{*}\right)(t)+S_{\lambda}\left(\psi^{*}\right)(t)+T_{\lambda}\left(\psi^{*}\right)(t)=f(t), \quad t \in\right] 0, \delta[, \\
u(0)=d(0)+n(0)+v(0)=\varphi^{*}+0+0=\varphi, \\
u^{\prime}(\delta)=v^{\prime}(\delta)+d^{\prime}(\delta)+n^{\prime}(\delta)=V_{\lambda}\left(f^{*}\right)+D_{\lambda}\left(\varphi^{*}\right)+\psi^{*}+N_{\lambda}\left(\psi^{*}\right)=\psi,
\end{array}\right.
$$

which can be written under the abstract form

$$
\left(\begin{array}{ccc}
I+P_{\lambda}+R_{\lambda} & F_{\lambda}+G_{\lambda} & S_{\lambda}+T_{\lambda} \\
0 & I & 0 \\
V_{\lambda} & D_{\lambda} & I+N_{\lambda}
\end{array}\right)\left(\begin{array}{l}
f^{*} \\
\varphi^{*} \\
\psi^{*}
\end{array}\right)=\left(\begin{array}{l}
f \\
\varphi \\
\psi
\end{array}\right) .
$$

We have necessarily $\varphi^{*}=\varphi$. It remains to solve, for $\lambda>0$, the system

$$
\left(\begin{array}{cc}
I+P_{\lambda}+R_{\lambda} & S_{\lambda}+T_{\lambda} \\
V_{\lambda} & I+N_{\lambda}
\end{array}\right)\left(\begin{array}{c}
f^{*} \\
\psi^{*}
\end{array}\right)\left(\begin{array}{c}
f-\left(F_{\lambda}+G_{\lambda}\right)(\varphi) \\
\psi-D_{\lambda}(\varphi)
\end{array}\right),
$$

that is,

$$
\left(I+A_{\lambda}\right) X=Y,
$$

with $A_{\lambda}$ defined as

$$
\begin{aligned}
& A_{\lambda}: \quad C(E) \times D_{q(0)}(1 / 2+\epsilon,+\infty) \quad \longrightarrow \quad C(E) \times E \\
& \left(f^{*}, \psi^{*}\right) \quad \longmapsto \quad\left(\begin{array}{cc}
P_{\lambda}+R_{\lambda} & S_{\lambda}+T_{\lambda} \\
V_{\lambda} & N_{\lambda}
\end{array}\right)\left(\begin{array}{l}
f^{*} \\
\psi^{*}
\end{array}\right) .
\end{aligned}
$$

This means that we have to inverse $I+A_{\lambda}$ in an appropriate normed space. We will choose the following norm:

$$
\begin{array}{r}
\left\|A_{\lambda}\right\|=\max \left\{\left\|P_{\lambda}+R_{\lambda}\right\|_{L(C(E))}, \quad\left\|S_{\lambda}+T_{\lambda}\right\|_{L\left(D_{q(0)}(1 / 2+\epsilon,+\infty), C(E)\right)},\right. \\
\left.\left\|V_{\lambda}\right\|_{L\left(C(E), D_{q(0)}(1 / 2+\epsilon,+\infty)\right)}, \quad\left\|N_{\lambda}\right\|_{L\left(D_{q(0)}(1 / 2+\epsilon,+\infty), E\right)}\right\}
\end{array}
$$

for the operator $A_{\lambda}$ of $L\left(C(E) \times D_{q(0)}(1 / 2+\epsilon,+\infty), C(E) \times E\right)$.

\section{Resolution of the system and regularity of $u$}

\subsection{Norm of the system matrix}

We recall that (see (13))

$$
\left\|\left(P_{\lambda}+R_{\lambda}\right)\right\|_{L(C([0, \delta] ; E))} \leq C\left(1 / \lambda^{\alpha+1 / 2}+1 / \lambda^{\rho}+1 / \lambda^{1 / 2}\right) .
$$


The other operators occurring in the system matrix are

$$
\begin{aligned}
S_{\lambda}\left(\psi^{*}\right)(t)= & \frac{1}{2 i \pi} \int_{\gamma} \frac{s \sqrt{-z}(t)}{\sqrt{-z}} \frac{\partial^{2}}{\partial t^{2}}(Q(t)-z I)^{-1} \psi^{*} d z \\
& +\frac{2}{2 i \pi} \int_{\gamma} c_{\sqrt{-z}}(\delta-t) \frac{\partial}{\partial t}(Q(t)-z I)^{-1} \psi^{*} d z \\
T_{\lambda}\left(\psi^{*}\right)(t)= & \frac{1}{2 i \pi} \int_{\gamma} \frac{s \sqrt{-z}(t)}{\sqrt{-z}} p(t) \frac{\partial}{\partial t}(Q(t)-z I)^{-1} \psi^{*} d z \\
& +\frac{1}{2 i \pi} \int_{\gamma} c_{\sqrt{-z}}(\delta-t) p(t)(Q(t)-z I)^{-1} \psi^{*} d z
\end{aligned}
$$

and the operators (independent of $t$ )

$$
\begin{aligned}
& V_{\lambda}\left(f^{*}\right)=-\left.\frac{1}{2 i \pi} \int_{\gamma} \int_{0}^{\delta} \frac{s \sqrt{-z}(\xi)}{\sqrt{-z}} \frac{\partial}{\partial t}(Q(t)-z I)^{-1}\right|_{t=\delta} f^{*}(\xi) d \xi d z, \\
& N_{\lambda}\left(\psi^{*}\right)=\left.\frac{1}{2 i \pi} \int_{\gamma} \frac{s_{\sqrt{-z}}(\delta)}{\sqrt{-z}} \frac{\partial}{\partial t}(Q(t)-z I)^{-1}\right|_{t=\delta} \psi^{*} d z .
\end{aligned}
$$

Step 1. $\quad V_{\lambda}$ and $N_{\lambda}$ fulfill the estimates

$$
\begin{aligned}
\left\|V_{\lambda}\left(f^{*}\right)\right\| & =\left\|-\left.\frac{1}{2 i \pi} \int_{\gamma} \int_{0}^{\delta} \frac{s \sqrt{-z}(\xi)}{\sqrt{-z}} \frac{\partial}{\partial t}(Q(t)-z I)^{-1}\right|_{t=\delta} f^{*}(\xi) d \xi d z\right\| \\
& \leq C \cdot \delta \cdot \int_{\gamma} \frac{1}{|z|^{1 / 2}|z+\lambda|^{\alpha+1 / 2}}|d z|\left\|f^{*}\right\|_{C(E)} \leq \frac{C \cdot \delta}{|\lambda|^{\alpha}}\left\|f^{*}\right\|_{C(E)}, \\
\left\|N_{\lambda}\left(\psi^{*}\right)\right\| & =\left\|\left.\frac{1}{2 i \pi} \int_{\gamma} \frac{s \sqrt{-z}(\delta)}{\sqrt{-z}} \frac{\partial}{\partial t}(Q(t)-z I)^{-1}\right|_{t=\delta} \psi^{*} d z\right\| \\
& \leq \frac{C}{|\lambda|^{\alpha}}\left\|\psi^{*}\right\|_{E} .
\end{aligned}
$$

Regarding $T_{\lambda}$, we have

$$
\begin{aligned}
\left\|T_{\lambda}\left(\psi^{*}\right)(t)\right\| \leq & \left\|\frac{1}{2 i \pi} \int_{\gamma} \frac{s_{\sqrt{-z}}(t)}{\sqrt{-z}} p(t) \frac{\partial}{\partial t}(Q(t)-z I)^{-1} \psi^{*} d z\right\| \\
& +\left\|\frac{1}{2 i \pi} \int_{\gamma} c_{\sqrt{-z}}(\delta-t) p(t)(Q(t)-z I)^{-1} \psi^{*} d z\right\| \\
\leq & \frac{C}{|\lambda|^{\alpha}}\left\|\psi^{*}\right\|_{E}+\left\|\frac{1}{2 i \pi} \int_{\gamma} c_{\sqrt{-z}}(\delta-t) p(t)(Q(t)-z I)^{-1} \psi^{*} d z\right\| .
\end{aligned}
$$


Observe that without more regularity on $\psi^{*}$, we cannot obtain an estimate of the previous integral, except in $C\left\|\psi^{*}\right\|_{E}$.

Step 2. In order to derive the suitable estimates for this term (then for $T_{\lambda}$, and also for $S_{\lambda}$ ) we consider the interpolation space in which $\psi^{*}$ lies, that is,

$$
D_{Q(\delta)}(1 / 2+\epsilon,+\infty)=D_{Q(0)}(1 / 2+\epsilon,+\infty)=D_{q(0)}(1 / 2+\epsilon,+\infty),
$$

and the natural assumption (14).

Let $\psi^{*} \in D_{Q(0)}(1 / 2+\epsilon,+\infty)$. The operators $p(t)$ being bounded, we have

$$
\begin{aligned}
\frac{1}{2 i \pi} \int_{\gamma} c_{\sqrt{-z}}(\delta-t) p(t)(Q(t)-z I)^{-1} \psi^{*} d z \\
=\frac{1}{2 i \pi} \int_{\gamma} c_{\sqrt{-z}}(\delta-t)\left(p(t)(Q(t)-z I)^{-1}-p(\delta)(Q(\delta)-z I)^{-1}\right) \psi^{*} d z \\
+\frac{1}{2 i \pi} \int_{\gamma} c_{\sqrt{-z}}(\delta-t) p(\delta)(Q(\delta)-z I)^{-1} \psi^{*} d z \\
=\frac{1}{2 i \pi} \int_{\gamma} c_{\sqrt{-z}}(\delta-t) p(t) \int_{\delta}^{t} \frac{\partial}{\partial r}(Q(r)-z I)^{-1} \psi^{*} d r d z \\
+\frac{1}{2 i \pi} \int_{\gamma} c_{\sqrt{-z}}(\delta-t)(p(t)-p(\delta)) \frac{Q(\delta)(Q(\delta)-z I)^{-1}}{z} \psi^{*} d z \\
+\frac{1}{2 i \pi} \int_{\gamma} c_{\sqrt{-z}}(\delta-t) p(\delta) \frac{Q(\delta)(Q(\delta)-z I)^{-1}}{z} \psi^{*} d z \\
\frac{1}{2 i \pi} \int_{\gamma} c_{\sqrt{-z}}(\delta-t) p(t)(Q(t)-z I)^{-1} \psi^{*} d z:=I_{1}+I_{2}+I_{3} .
\end{aligned}
$$

Step 3. Regarding $I_{1}$, let $I_{1}:=J_{1}^{+}+J_{1}^{-}$where $J_{1}^{+}$and $J_{1}^{-}$denote respectively the integrals on the parts $\gamma_{+}=\left\{z \in \gamma:|z| \geq 1 /(\delta-t)^{2}\right\}$ and $\gamma_{-}=\{z \in \gamma:|z| \leq$ $\left.1 /(\delta-t)^{2}\right\}$ of $\gamma$. Hereafter we will estimate $J_{1}^{+}$; the same holds for $J_{1}^{-}$.

$$
\left\|J_{1}^{+}\right\| \leq C \int_{\gamma_{+}}\left\|c_{s q r t-z}(\delta-t) \int_{\delta}^{t} \frac{\partial}{\partial r}(Q(r)-z I)^{-1} \psi^{*} d r\right\||d z| .
$$

In the sequel, we will use the following estimates:

$$
\exists C>0: \forall z \in \gamma, \forall \lambda>0 \quad|z+\lambda| \geq C \lambda \quad \text { and } \quad|z+\lambda| \geq C|z| .
$$


It follows that

$$
\begin{aligned}
\left\|J_{1}^{+}\right\| & \leq C \int_{\gamma_{+}}\left\|c_{\sqrt{-z}}(\delta-t) \int_{\delta}^{t} \frac{\partial}{\partial r}(Q(r)-z I)^{-1} \psi^{*} d r\right\||d z| \\
& \leq C \int_{\gamma_{+}} e^{-|z|^{1 / 2}(\delta-t)} \frac{(\delta-t)}{\lambda^{\alpha}|z|^{1 / 2}}|d z|\left\|\psi^{*}\right\|_{E} \\
& \leq \frac{C}{\lambda^{\alpha}} \int_{1}^{\infty} e^{-\sigma} \frac{(\delta-t)}{\left(\frac{\sigma^{2}}{(\delta-t)^{2}}\right)^{1 / 2}} \frac{\sigma d \sigma}{(\delta-t)^{2}}\left\|\psi^{*}\right\|_{E} \\
& \leq \frac{C}{\lambda^{\alpha}}\left\|\psi^{*}\right\|_{E} .
\end{aligned}
$$

For $I_{3}$, we have (the same holds true for $I_{2}$ )

$$
\begin{aligned}
\left\|I_{3}\right\| & =\left\|\frac{1}{2 i \pi} \int_{\gamma} c_{\sqrt{-z}}(\delta-t) p(\delta) \frac{Q(\delta)(Q(\delta)-z I)^{-1}}{z} \psi^{*} d z\right\| \\
& \leq C \int_{\gamma} \frac{1}{|z \| z+\lambda|^{1 / 2+\epsilon}}|d z|\left\|\psi^{*}\right\|_{D_{Q(0)}(1 / 2+\epsilon,+\infty)} \\
& \leq \frac{C}{\lambda^{1 / 2+\epsilon}}\left\|\psi^{*}\right\|_{D_{Q(0)}(1 / 2+\epsilon,+\infty)} .
\end{aligned}
$$

Step 4. Finally, we have to estimate the operator $S_{\lambda}$. It is the most singular since it contains the second derivative of the resolvent $Q(\cdot)$. We have

$$
\begin{aligned}
S_{\lambda}\left(\psi^{*}\right)(t)= & \frac{1}{2 i \pi} \int_{\gamma} \frac{s \sqrt{-z}(t)}{\sqrt{-z}} \frac{\partial^{2}}{\partial t^{2}}(Q(t)-z I)^{-1} \psi^{*} d z \\
& +\frac{2}{2 i \pi} \int_{\gamma} c_{\sqrt{-z}}(\delta-t) \frac{\partial}{\partial t}(Q(t)-z I)^{-1} \psi^{*} d z, \\
S_{\lambda}\left(\psi^{*}\right)(t):= & l_{1}(t)+l_{2}(t) .
\end{aligned}
$$

In what follows, we will only estimate the integral $l_{1}$. (For the second one, $l_{2}$, the techniques are similar.) First of all, write

$$
\begin{aligned}
l_{1}= & \frac{1}{2 i \pi} \int_{\gamma} \frac{s \sqrt{-z}(t)}{\sqrt{-z}} \frac{\partial^{2}}{\partial t^{2}}(Q(t)-z I)^{-1} \psi^{*} d z \\
= & \frac{1}{2 i \pi} \int_{\gamma} \frac{s \sqrt{-z}(t)}{\sqrt{-z}}\left(\frac{\partial^{2}}{\partial t^{2}}(Q(t)-z I)^{-1}-\frac{\partial^{2}}{\partial t^{2}}(Q(t)-z I)_{\left.\right|_{t=\delta} ^{-1}}^{-1}\right) \psi^{*} d z \\
& +\frac{1}{2 i \pi} \int_{\gamma} \frac{s \sqrt{-z}(t)}{\sqrt{-z}} \frac{\partial^{2}}{\partial t^{2}}(Q(t)-z I)_{\left.\right|_{t=\delta} ^{-1}} \psi^{*} d z \\
:= & j_{1}+j_{2},
\end{aligned}
$$


and, as usual, let $j_{1}:=j_{1}^{+}+j_{1}^{-}$on $\gamma_{+}$and $\gamma_{-}$respectively. For the first integral $j_{1}^{+}$, we deduce

$$
\begin{aligned}
\left\|j_{1}^{+}\right\| & =C\left\|\int_{\gamma_{+}} \frac{s \sqrt{-z}(t)}{\sqrt{-z}}\left(\frac{\partial^{2}}{\partial t^{2}}(Q(t)-z I)^{-1}-\frac{\partial^{2}}{\partial t^{2}}(Q(t)-z I)_{\mid t=\delta}^{-1}\right) \psi^{*} d z\right\| \\
& \leq C \int_{\gamma_{+}} \frac{1}{|z|^{1 / 2}} e^{-|z|^{1 / 2}(\delta-t)} \frac{(\delta-t)^{2 \eta}}{|z+\lambda|^{\rho}}|d z|\left\|\psi^{*}\right\|_{E} \\
& \leq C \int_{\gamma_{+}} \frac{1}{|z|^{1 / 2}} e^{-|z|^{1 / 2}(\delta-t)} \frac{(\delta-t)^{2 \eta}}{|z+\lambda|^{\rho+\eta-1 / 2}|z+\lambda|^{1 / 2-\eta}}|d z|\left\|\psi^{*}\right\|_{E} \\
& \leq \frac{C}{\lambda^{\rho+\eta-1 / 2}} \int_{\gamma_{+}} \frac{1}{|z|^{1 / 2}} e^{-|z|^{1 / 2}(\delta-t)} \frac{(\delta-t)^{2 \eta}}{|z|^{1 / 2-\eta}}|d z|\left\|\psi^{*}\right\|_{E} \\
& \leq \frac{C}{\lambda^{\rho+\eta-1 / 2}} \int_{1}^{\infty} e^{-\sigma} \frac{(\delta-t)^{2 \eta}}{\left(\frac{\sigma^{2}}{(\delta-t)^{2}}\right)^{1-\eta}} \frac{\sigma d \sigma}{(\delta-t)^{2}}\left\|\psi^{*}\right\|_{E} \\
& \leq \frac{C}{\lambda^{\rho+\eta-1 / 2}}\left\|\psi^{*}\right\|_{E} .
\end{aligned}
$$

A similar estimate holds true for $j_{1}^{-}$.

Step 5. Regarding $j_{2}$, we make use of the following identity (already mentioned)

$$
\begin{aligned}
\frac{\partial^{2}}{\partial t^{2}}(Q(t)-z I)_{\mid t=\delta}^{-1} \psi^{*} & =Q(\delta)(Q(\delta)-z I)^{-1} \frac{\partial^{2}}{\partial t^{2}} Q(t)_{\left.\right|_{t=\delta} ^{-1}}^{-1} Q(\delta)(Q(\delta)-z I)^{-1} \psi^{*} \\
+ & 2 z\left(Q(\delta)(Q(\delta)-z I)^{-1} \frac{\partial}{\partial t} Q(t)_{\mid t=\delta}^{-1}\right)^{2} Q(\delta)(Q(\delta)-z I)^{-1} \psi^{*} .
\end{aligned}
$$

Hence

$$
\begin{aligned}
j_{2}= & \frac{1}{2 i \pi} \int_{\gamma} \frac{s \sqrt{-z}(t)}{\sqrt{-z}} Q(\delta)(Q(\delta)-z I)^{-1} \frac{\partial^{2}}{\partial t^{2}} Q(t)_{\mid t=\delta}^{-1} Q(\delta)(Q(\delta)-z I)^{-1} \psi^{*} d z \\
& +\frac{1}{2 i \pi} \int_{\gamma} \frac{2 z s \sqrt{-z}}{\sqrt{-z}}(t) \\
j_{2}:= & a_{1}+a_{2},
\end{aligned}
$$

$$
\begin{aligned}
\left\|a_{1}\right\| & \leq C \int_{\gamma} \frac{1}{|z|^{1 / 2}|z+\lambda|^{1 / 2+\epsilon}}|d z|\left\|\psi^{*}\right\|_{D_{Q(0)}(1 / 2+\epsilon,+\infty)} \\
& \leq \frac{C}{\lambda^{\epsilon}}\left\|\psi^{*}\right\|_{D_{Q(0)}(1 / 2+\epsilon,+\infty)},
\end{aligned}
$$


and

$$
\begin{aligned}
\left\|a_{2}\right\| & \leq C \int_{\gamma} \frac{|z|}{|z|^{1 / 2}|z+\lambda|^{2 \alpha+1+\epsilon+1 / 2}}|d z|\left\|\psi^{*}\right\|_{D_{Q(0)}(1 / 2+\epsilon,+\infty)} \\
& \leq C \int_{\gamma} \frac{1}{|z+\lambda|^{2 \alpha+1+\epsilon}}|d z|\left\|\psi^{*}\right\|_{D_{Q(0)}(1 / 2+\epsilon,+\infty)} \\
& \leq \frac{C}{\lambda^{2 \alpha+\epsilon}}\left\|\psi^{*}\right\|_{D_{Q(0)}(1 / 2+\epsilon,+\infty)} .
\end{aligned}
$$

Summarizing, we can make $\left\|A_{\lambda}\right\|$ small enough and deduce the following result about the resolution of the system.

Theorem 2.1. Under the assumptions (6)-(9) and

$$
f \in C([0, \delta] ; E), \quad \psi \in D_{q(0)}(1 / 2+\epsilon,+\infty), \quad \varphi \in D(q(0)),
$$

there exists $\lambda^{*}>0$ such that for all $\lambda \geq \lambda^{*}$, the operator $I+A_{\lambda}$ is invertible, i.e., the system

$$
\left(\begin{array}{ll}
I & 0 \\
0 & I
\end{array}\right)\left(\begin{array}{c}
f^{*} \\
\psi^{*}
\end{array}\right)+\left(\begin{array}{cc}
P_{\lambda}+R_{\lambda} & S_{\lambda}+T_{\lambda} \\
V_{\lambda} & N_{\lambda}
\end{array}\right)\left(\begin{array}{c}
f^{*} \\
\psi^{*}
\end{array}\right)=\left(\begin{array}{c}
f-\left(F_{\lambda}+G_{\lambda}\right)(\varphi) \\
\psi-D_{\lambda}(\varphi)
\end{array}\right),
$$

admits a unique solution $\left(f^{*}, \psi^{*}\right) \in C([0, \delta] ; E) \times D_{q(0)}(1 / 2+\epsilon,+\infty)$.

\subsection{Regularity of the solution}

Since the term

$$
D_{\lambda}(\varphi)=\left.\frac{1}{2 i \pi} \int_{\gamma} \frac{1}{\cosh \sqrt{-z} \delta} \frac{\partial}{\partial t}(Q(t)-z I)^{-1}\right|_{t=\delta} \varphi d z,
$$

is very regular (thanks to $\cosh \sqrt{-z} \delta$ ) and

$$
\psi \in D_{q(0)}(1 / 2+\epsilon,+\infty)=D_{q(0)}(1 / 2+\epsilon,+\infty),
$$

then

$$
\psi-D_{\lambda}(\varphi) \in D_{q(\delta)}(1 / 2+\epsilon,+\infty)=D_{q(0)}(1 / 2+\epsilon,+\infty) .
$$

So, $A_{\lambda}$ takes its values in $C(E) \times D_{q(0)}(1 / 2+\epsilon,+\infty)$. The first regularity result is the following

Proposition 2.2. Under the assumptions (6)-(9) and

$$
f \in C^{2 \theta}([0, \delta] ; E), \quad \psi \in D_{q(0)}(1 / 2+\epsilon,+\infty), \quad \varphi \in D(q(0)),
$$

there exists $\lambda^{*}>0$ such that for all $\lambda \geq \lambda^{*}$, the function $f^{*}$ is in the space

$$
C^{\beta}([0, \delta] ; E),
$$

where $\beta=\min (2 \alpha, 2 \theta, 2 \eta+2 \rho-1)$. 
Proof. Let $\lambda \geq \lambda^{*}$, One has

$$
f^{*}=f-\left(P_{\lambda}+R_{\lambda}\right)\left(f^{*}\right)-\left(F_{\lambda}+G_{\lambda}\right)(\varphi)-\left(S_{\lambda}+T_{\lambda}\right)\left(\psi^{*}\right) .
$$

Using the same techniques as in [9, p. 99, Proposition 4.3] the term $\left(P_{\lambda}+R_{\lambda}\right)\left(f^{*}\right)$ (which is not easy to handle) is Hölderian of exponent

$$
\min (2 \alpha, 2 \eta+2 \rho-1)
$$

while (due to our previous calculations), the functions

$$
F_{\lambda}(\varphi), \quad G_{\lambda}(\varphi), \quad S_{\lambda}\left(\psi^{*}\right), \quad T_{\lambda}\left(\psi^{*}\right)
$$

are Hölderian of exponent

$$
\min (2 \alpha+2 \epsilon, 2 \eta+2 \rho-1)
$$

The terms $T_{\lambda}\left(\psi^{*}\right), G_{\lambda}(\varphi)$ are not too hard because they correspond to the those containing the perturbation $p(t)$.

In order to give an optimal regularity result of the solution, we need the following (important) lemma

Lemma 2.3. Under the assumptions (6)-(9) and

$$
\psi \in D_{q(0)}(1 / 2+\epsilon,+\infty), \quad \varphi \in D(q(0))
$$

one has

$$
f^{*}(0)=f(0)-\left.\left(\frac{d^{2}}{d t^{2}} Q(t)^{-1}\right)\right|_{t=0} Q(0) \varphi+r_{\delta}\left(\varphi, \psi^{*}, f^{*}\right)
$$

where $r_{\delta}\left(\varphi, \psi^{*}, f^{*}\right) \in D_{q(0)}(\alpha,+\infty)=D_{Q(0)}(\alpha,+\infty)$.

Proof. Recall that

$$
f^{*}(0)=f(0)-\left(P_{\lambda}+R_{\lambda}\right)\left(f^{*}\right)(0)-\left(F_{\lambda}+G_{\lambda}\right)(\varphi)(0)-\left(S_{\lambda}+T_{\lambda}\right)\left(\psi^{*}\right)(0),
$$


where

$$
\begin{aligned}
P_{\lambda}\left(f^{*}\right)(0)= & -\left.\frac{1}{i \pi} \int_{\gamma} \int_{0}^{\delta} c_{\sqrt{-z}}(s) \frac{\partial}{\partial t}(Q(t)-z I)^{-1}\right|_{t=0} f^{*}(s) d s d z \\
R_{\lambda}\left(f^{*}\right)(0)= & -\left.\frac{1}{2 i \pi} \int_{\gamma} \int_{0}^{\delta} c_{\sqrt{-z}}(s) p(0)(Q(0)-z I)^{-1}\right|_{t=0} f^{*}(s) d s d z, \\
F_{\lambda}(\varphi)(0)= & \lim _{t \rightarrow 0}\left(\frac{1}{2 i \pi} \int_{\gamma} c_{\sqrt{-z}}(t) \frac{\partial^{2}}{\partial t^{2}}(Q(t)-z I)^{-1} \varphi d z\right) \\
& -\left.\frac{1}{i \pi} \int_{\gamma} \sqrt{-z} s_{\sqrt{-z}}(\delta) \frac{\partial}{\partial t}(Q(t)-z I)^{-1}\right|_{t=0} \varphi d z \\
F_{\lambda}(\varphi)(0)= & F_{\lambda}^{1}(\varphi)(0)+F_{\lambda}^{2}(\varphi)(0) \\
G_{\lambda}(\varphi)(0)= & \frac{-1}{2 i \pi} \int_{\gamma} \sqrt{-z} s_{\sqrt{-z}}(\delta) \frac{p(0)(Q(0)-z I)^{-1} Q(0) \varphi}{z} d z
\end{aligned}
$$

and

$$
\begin{aligned}
& S_{\lambda}\left(\psi^{*}\right)(0)=\left.\frac{1}{i \pi} \int_{\gamma} \frac{1}{\cosh \sqrt{-z} \delta} \frac{\partial}{\partial t}(Q(t)-z I)^{-1}\right|_{t=0} \psi^{*} d z \\
& T_{\lambda}\left(\psi^{*}\right)(0)=\frac{1}{2 i \pi} \int_{\gamma} \frac{\left.p(0)(Q(0)-z I)^{-1}\right|_{t=0} \psi^{*}}{\cosh \sqrt{-z} \delta} d z .
\end{aligned}
$$

Let us treat the regularity of each term.

Step 1. Recall that (for $r>0$ )

$$
\begin{aligned}
& Q(0)(Q(0)-r)^{-1} \frac{\partial}{\partial t}(Q(t)-z I)_{\mid t=0}^{-1} \varphi \\
& =Q(0)(Q(0)-r)^{-1} Q(0)(Q(0)-z)^{-1} \frac{d}{d t}(Q(t))_{\mid t=0}^{-1} Q(0)(Q(0)-z I)^{-1} \varphi,
\end{aligned}
$$

and

$$
\begin{aligned}
Q(0)(Q(0)-r)^{-1} Q(0)(Q(0) & -z)^{-1} \\
& =\frac{z}{r-z} Q(0)(Q(0)-z)^{-1}-\frac{r}{r-z} Q(0)(Q(0)-r)^{-1} .
\end{aligned}
$$


Hence

$$
\begin{aligned}
Q(0)(Q(0) & -r)^{-1} P_{\lambda}\left(f^{*}\right)(0) \\
=- & \frac{1}{i \pi} \int_{\gamma} \int_{0}^{\delta} c_{\sqrt{-z}}(s) \frac{z}{r-z} \\
& \times\left\{Q(0)(Q(0)-z)^{-1} \frac{d}{d t}(Q(t))_{\mid t=0}^{-1} Q(0)(Q(0)-z I)^{-1} f^{*}(s)\right\} d s d z \\
+ & \frac{1}{i \pi} \int_{\gamma} \int_{0}^{\delta} c_{\sqrt{-z}}(s) \frac{r}{r-z} \\
& \times\left\{Q(0)(Q(0)-r)^{-1} \frac{d}{d t}(Q(t))_{\mid t=0}^{-1} Q(0)(Q(0)-z I)^{-1} f^{*}(s)\right\} d s d z \\
Q(0)(Q(0) & -r)^{-1} P_{\lambda}\left(f^{*}\right)(0):=(\mathrm{I})+(\mathrm{II}) .
\end{aligned}
$$

Note that

$$
\begin{aligned}
\|(\mathrm{I})\| & \leq C \int_{\gamma} \frac{|z|}{|r-z|} \frac{1}{|z|^{\alpha+1 / 2}}\left(\int_{0}^{\delta} e^{-s|z|^{1 / 2}} d s\right) d|z|\left\|f^{*}\right\|_{C([0, \delta] ; E)} \\
& \leq C \int_{\gamma} \frac{|z|}{|r-z||z|^{\alpha+1}}\left(\int_{0}^{\infty} e^{-\xi} d \xi\right) d|z|\left\|f^{*}\right\|_{C([0, \delta] ; E)} \\
& \leq \frac{C}{r^{\alpha}}\left\|f^{*}\right\|_{C([0, \delta] ; E)}
\end{aligned}
$$

and we have a similar estimate for (II). Then $P_{\lambda}\left(f^{*}\right)(0) \in D_{q(0)}(\alpha,+\infty)$. It is clear that the term $R_{\lambda}\left(f^{*}\right)(0)$, (which is more regular than $P_{\lambda}\left(f^{*}\right)(0)$ due to the perturbation), is also in $D_{q(0)}(\alpha,+\infty)$.

Step 2. We have

$$
\begin{aligned}
F_{\lambda}^{2}(\varphi)(0)=-\frac{1}{i \pi} \int_{\gamma} & \sqrt{-z} s_{\sqrt{-z}}(\delta) \\
& \times\left\{Q(0)(Q(0)-z)^{-1} \frac{d}{d t}(Q(t))_{\mid t=0}^{-1}(Q(0)-z I)^{-1} Q(0) \varphi\right\} d z
\end{aligned}
$$

and

$$
\begin{aligned}
Q(0) & (Q(0)-r)^{-1} F_{\lambda}(\varphi)(0) \\
=- & \frac{1}{i \pi} \int_{\gamma} \sqrt{-z} s_{\sqrt{-z}}(\delta) \frac{z}{r-z} \\
& \times\left\{Q(0)(Q(0)-z)^{-1} \frac{d}{d t}(Q(t))_{\mid t=0}^{-1}(Q(0)-z I)^{-1} Q(0) \varphi\right\} d z \\
+ & \frac{1}{i \pi} \int_{\gamma} \sqrt{-z} s_{\sqrt{-z}}(\delta) \frac{r}{r-z} \\
& \times\left\{Q(0)(Q(0)-r)^{-1} \frac{d}{d t}(Q(t))_{\mid t=0}^{-1}(Q(0)-z I)^{-1} Q(0) \varphi\right\} d z,
\end{aligned}
$$




$$
\begin{gathered}
Q(0)(Q(0)-r)^{-1} F_{\lambda}(\varphi)(0):=(\mathrm{I})+(\mathrm{II}), \\
\|(\mathrm{I})\| \leq C \int_{\gamma} \frac{|z|^{1+1 / 2}}{|r-z|} \frac{1}{|z|^{\alpha+1+1 / 2}} d|z|\|Q(0) \varphi\|_{E} \leq \frac{C}{r^{\alpha}}\|Q(0) \varphi\|_{E} .
\end{gathered}
$$

A similar estimate holds true for (II).

For $G_{\lambda}(\varphi)(0)$, similar estimates give

$$
G_{\lambda}(\varphi)(0) \in D_{q(0)}(1 / 2,+\infty) \subset D_{q(0)}(\alpha,+\infty)
$$

since $\alpha \leq 1 / 2$.

Step 3. Regarding $F_{\lambda}^{1}(\varphi)(0)$, before considering the second derivative with respect to $t$, write

$$
\begin{aligned}
&(Q(t)-z I)^{-1} \varphi \\
&=\frac{Q(t)(Q(t)-z I)^{-1} \varphi}{z}-\frac{\varphi}{z} \\
&=\frac{Q(t)(Q(t)-z I)^{-1}}{z}\left(Q(0)^{-1}-Q(t)^{-1}+t \frac{d}{d t} Q(t)^{-1}\right) Q(0) \varphi-\frac{\varphi}{z} \\
&+\frac{(Q(t)-z I)^{-1}}{z} Q(0) \varphi-t \cdot \frac{Q(t)(Q(t)-z I)^{-1}}{z} \frac{d}{d t} Q(t)^{-1} Q(0) \varphi \\
&= \frac{1}{z}\left(Q(0)^{-1}-Q(t)^{-1}+t \frac{\partial}{\partial t} Q(t)^{-1}\right) Q(0) \varphi-\frac{\varphi}{z}-\frac{t}{z} \frac{d}{d t} Q(t)^{-1} Q(0) \varphi \\
&+(Q(t)-z I)^{-1}\left(Q(0)^{-1}-Q(t)^{-1}+t \frac{d}{d t} Q(t)^{-1}\right) Q(0) \varphi \\
&+\frac{(Q(t)-z I)^{-1}}{z} Q(0) \varphi-t \cdot(Q(t)-z I)^{-1} \frac{d}{d t} Q(t)^{-1} Q(0) \varphi .
\end{aligned}
$$

Now, we apply the second-differentiation operator on the resolvent in the previous presentation. Using Dunford calculus, we find

$$
\begin{aligned}
\frac{1}{2 i \pi} \int_{\gamma} c_{\sqrt{-z}}(t) \frac{\partial^{2}}{\partial t^{2}}(Q(t)-z I)^{-1} \varphi d z \\
=\frac{1}{2 i \pi} \int_{\gamma} c_{\sqrt{-z}}(t) \frac{\partial^{2}}{\partial t^{2}}(Q(t)-z I)^{-1}\left(Q(0)^{-1}-Q(t)^{-1}+t \frac{d}{d t} Q(t)^{-1}\right) Q(0) \varphi d z \\
\quad+\frac{1}{2 i \pi} \int_{\gamma} c_{\sqrt{-z}}(t) \frac{1}{z} \frac{\partial^{2}}{\partial t^{2}}(Q(t)-z I)^{-1} Q(0) \varphi d z \\
\quad-\frac{1}{2 i \pi} \int_{\gamma} c_{\sqrt{-z}}(t) \frac{\partial^{2}}{\partial t^{2}}(Q(t)-z I)^{-1} \cdot t \cdot\left(\frac{d}{d t} Q(t)^{-1}\right) Q(0) \varphi d z \\
-\frac{1}{i \pi} \int_{\gamma} c_{\sqrt{-z}}(t) \frac{\partial}{\partial t}(Q(t)-z I)^{-1}\left(\frac{d}{d t} Q(t)^{-1}\right) Q(0) \varphi d z \\
\quad-\frac{1}{2 i \pi} \int_{\gamma} c_{\sqrt{-z}}(t)(Q(t)-z I)^{-1}\left(\frac{d^{2}}{d t^{2}} Q(t)^{-1}\right) Q(0) \varphi d z
\end{aligned}
$$




$$
\frac{1}{2 i \pi} \int_{\gamma} c_{\sqrt{-z}}(t) \frac{\partial^{2}}{\partial t^{2}}(Q(t)-z I)^{-1} \varphi d z:=S_{\delta}^{1}(t)+S_{\delta}^{2}(t)+S_{\delta}^{3}(t)+S_{\delta}^{4}(t)+S_{\delta}^{5}(t) .
$$

Step 4. Concerning the first term $S_{\delta}^{1}(t)$, observe that

$$
\begin{aligned}
\|\left(Q(0)^{-1}-Q(t)^{-1}+t \frac{d}{d t} Q(t)^{-1}\right) & Q(0) \varphi \| \\
& =\left\|-\int_{0}^{t}\left(\frac{d}{d \xi} Q(\xi)^{-1}-\frac{d}{d t} Q(t)^{-1}\right) Q(0) \varphi d \xi\right\| \\
& \leq C \int_{0}^{t}(t-\xi)^{2 \eta} d \xi\|Q(0) \varphi\| \leq C t^{2 \eta+1}\|Q(0) \varphi\|,
\end{aligned}
$$

and then, dividing the integral upon $\gamma$ into two parts as in the previous sections, we get, for instance, on the first part of $\gamma$,

$$
\begin{aligned}
\left\|S_{\delta}^{1}(t)\right\| & \leq C t^{2 \eta+1}\left(\int_{\gamma,|z| \geq \frac{1}{t^{2}}} e^{-t|z|^{1 / 2} \sin \left(\theta_{0} / 2\right)} \frac{1}{|z|^{\rho}}|d z|\right)\|Q(0) \varphi\| \\
& \leq C t^{2 \eta+1}\left(\int_{1}^{+\infty} e^{-\sigma} \frac{2 \sigma}{t^{2}\left(\frac{\sigma^{2}}{t^{2}}\right)^{\rho}} d \sigma\right)\|Q(0) \varphi\| \\
& \leq C t^{2 \rho+2 \eta-1}\|Q(0) \varphi\|,
\end{aligned}
$$

with a similar estimate on the second part of the curve. Therefore

$$
S_{\delta}^{1}(t) \rightarrow 0 \quad \text { as } t \rightarrow 0 .
$$

On the other hand, it is easy to see that

$$
\left\|c_{\sqrt{-z}}(t) \frac{1}{z} \frac{\partial^{2}}{\partial t^{2}}(Q(t)-z I)^{-1}\right\|=O\left(1 /|z|^{1+\rho}\right),
$$

then $S_{\delta}^{2}(t) \rightarrow 0$ as $t \rightarrow 0$.

- Regarding the third term $S_{\delta}^{3}(t)$, we use the formula

$$
\begin{aligned}
\frac{\partial^{2}}{\partial t^{2}}(Q(t)-z I)^{-1}= & Q(t)(Q(t)-z I)^{-1}\left(\frac{d^{2}}{d t^{2}} Q(t)^{-1}\right) Q(t)(Q(t)-z I)^{-1} \\
& +2 z Q(t)(Q(t)-z I)^{-1}\left(\frac{d Q(t)^{-1}}{d t} Q(t)(Q(t)-z I)^{-1}\right)^{2}
\end{aligned}
$$

and deduce

$$
\left\|t \frac{\partial^{2}}{\partial t^{2}}(Q(t)-z I)^{-1}\left(\frac{d}{d t} Q(t)^{-1}\right) Q(0) \varphi\right\|=O\left(t /|z|^{\alpha+1 / 2}\right)\|Q(0) \varphi\|,
$$

which leads to $\left\|S_{\delta}^{3}(t)\right\|=O\left(t^{2 \alpha}\right)\|Q(0) \varphi\| \rightarrow 0$ as $t \rightarrow 0$. 
- For the fourth term $S_{\delta}^{4}(t)$, note that (in virtue of assumption (8)

$$
\begin{aligned}
\| \frac{\partial}{\partial t}(Q(t)-z I)^{-1}\left(\frac{d}{d t} Q(t)^{-1}\right) & Q(0) \varphi \| \\
= & \left\|\left[Q(t)(Q(t)-z I)^{-1}\left(\frac{d}{d t} Q(t)^{-1}\right)\right]^{2} Q(0) \varphi\right\| \\
= & O\left(1 /|z|^{2 \alpha+1}\right)\|Q(0) \varphi\| .
\end{aligned}
$$

Consequently, integrating upon $\gamma$, we infer that

$$
\left\|S_{\delta}^{4}(t)\right\|=O\left(t^{4 \alpha-1}\right)\|Q(0) \varphi\| \rightarrow 0 \quad \text { as } t \rightarrow 0
$$

since $\alpha>1 / 4$.

- Finally, write

$$
\begin{aligned}
S_{\delta}^{5}(t)= & -\frac{1}{2 i \pi} \int_{\gamma} c_{\sqrt{-z}}(t)(Q(t)-z I)^{-1} \\
& \times\left(\left(\frac{d^{2}}{d t^{2}} Q(t)^{-1}\right)-\left.\left(\frac{d^{2}}{d t^{2}} Q(t)^{-1}\right)\right|_{t=0}\right) Q(0) \varphi d z \\
& -\frac{1}{2 i \pi} \int_{\gamma} c_{\sqrt{-z}}(t)\left((Q(t)-z I)^{-1}-(Q(0)-z I)^{-1}\right) \\
& \times\left.\left(\frac{d^{2}}{d t^{2}} Q(t)^{-1}\right)\right|_{t=0} Q(0) \varphi d z \\
& -\left.\frac{1}{2 i \pi} \int_{\gamma} c_{\sqrt{-z}}(t)(Q(0)-z I)^{-1}\left(\frac{d^{2}}{d t^{2}} Q(t)^{-1}\right)\right|_{t=0} Q(0) \varphi d z \\
S_{\delta}^{5}(t):= & S_{\delta}^{5,1}(t)+S_{\delta}^{5,2}(t)+S_{\delta}^{5,3}(t) .
\end{aligned}
$$

Then, as $t \rightarrow 0, S_{\delta}^{5,1}(t)$ and $S_{\delta}^{5,2}(t)$ tend to zero, while $S_{\delta}^{5,3}(t)$ tends to

$$
-\left.\left(\frac{d^{2}}{d t^{2}} Q(t)^{-1}\right)\right|_{t=0} Q(0) \varphi
$$

if and only if

$$
\left.\left(\frac{d^{2}}{d t^{2}} Q(t)^{-1}\right)\right|_{t=0} Q(0) \varphi \in \overline{D(Q(0))}
$$

due to Lemma 1.2.

Summing up, we obtain

$$
f^{*}(0)=f(0)-\left.\left(\frac{d^{2}}{d t^{2}} Q(t)^{-1}\right)\right|_{t=0} Q(0) \varphi+r_{\delta}\left(\varphi, \psi^{*}, f^{*}\right)
$$


where $r_{\delta}\left(\varphi, \psi^{*}, f^{*}\right) \in D_{q(0)}(\alpha,+\infty)=D_{Q(0)}(\alpha,+\infty)$. This completes the proof.

Theorem 2.4. Let $\varphi \in D(q(0)), \psi \in D_{q(0)}(1 / 2+\epsilon,+\infty)$ and $f \in C^{2 \theta}([0, \delta] ; E)$. Then, under the assumptions (6)-(9) and the compatibility condition

$$
f(0)-Q(0) \varphi-\left.\frac{d^{2} Q(t)^{-1}}{d t^{2}}\right|_{t=0} \varphi \in D_{q(0)}(\theta,+\infty),
$$

if $\lambda \geq \lambda^{*}$, the function u given in the representation (10) is the unique strict solution of Problem (2) satisfying the regularity

$$
u^{\prime \prime}, p(\cdot) u^{\prime}, q(\cdot) u(\cdot) \in C^{\beta}([0, \delta] ; E)
$$

where $\beta=\min (2 \alpha, 2 \theta, 2 \rho+2 \eta-1)$.

Proof. Recall that

$$
u(t)=d\left(t, Q((t)) \varphi^{*}+n\left(t, Q((t)) \psi^{*}+v\left(t, Q(t), f^{*}\right) .\right.\right.
$$

It remains to prove that

$$
u^{\prime \prime}, p(\cdot) u^{\prime}, q(\cdot) u(\cdot) \in C^{\beta}([0, \delta] ; E) .
$$

Step 1. Let us treat, for instance, the term $q(\cdot) u(\cdot)$. One has

$$
\begin{aligned}
Q(t) u(t)= & Q(t) v(t)+Q(t) d(t, Q(t)) \varphi+Q(t) n(t, Q(t)) \psi^{*} \\
= & -\frac{1}{2 i \pi} \int_{\gamma} \int_{0}^{\delta} K_{\sqrt{-z}}(t, s) Q(t)(Q(t)-z I)^{-1}\left(f^{*}(s)-f^{*}(t)\right) d s d z \\
& -\frac{1}{2 i \pi} \int_{\gamma} c_{\sqrt{-z}}(t)(Q(t)-z I)^{-1}\left(f^{*}(t)-f^{*}(0)\right) d z+f^{*}(t) \\
& -\frac{1}{2 i \pi} \int_{\gamma} c_{\sqrt{-z}}(t)(Q(t)-z I)^{-1}\left(f^{*}(0)\right) d z \\
& +\frac{1}{2 i \pi} \int_{\gamma} c_{\sqrt{-z}}(t) Q(t)(Q(t)-z I)^{-1} \varphi d z \\
& +\frac{1}{2 i \pi} \int_{\gamma} \frac{s_{\sqrt{-z}}(t)}{\sqrt{-z}} Q(t)(Q(t)-z I)^{-1} \psi^{*} d z \\
Q(t) u(t):= & \sum_{i=1}^{6} \Delta_{i}(t) .
\end{aligned}
$$

Thanks to Da Prato-Grisvard [6], the three first terms are Hölderian since the function $g^{*}$ defined by $g^{*}(t)=f^{*}(t)-f^{*}(0)$ is Hölderian and $g^{*}(0)=0$. 
Step 2. Regarding the terms $\Delta_{5}(t)$ and $\Delta_{6}(t)$, we have

$$
\begin{aligned}
\Delta_{5}(t)+\Delta_{6}(t)= & -\frac{1}{2 i \pi} \int_{\gamma} c_{\sqrt{-z}}(t)(Q(t)-z I)^{-1}\left(f^{*}(0)\right) d z \\
& +\frac{1}{2 i \pi} \int_{\gamma} c_{\sqrt{-z}}(t) Q(t)(Q(t)-z I)^{-1} \varphi d z \\
= & -\frac{1}{2 i \pi} \int_{\gamma} c_{\sqrt{-z}}(t)(Q(t)-z I)^{-1}\left(f^{*}(0)\right) d z \\
& +\frac{1}{2 i \pi} \int_{\gamma} c_{\sqrt{-z}}(t) Q(t)(Q(t)-z I)^{-1}\left(Q(0)^{-1}-Q(t)^{-1}\right) Q(0) \varphi d z \\
& +\frac{1}{2 i \pi} \int_{\gamma} c_{\sqrt{-z}}(t)(Q(t)-z I)^{-1} Q(0) \varphi d z \\
= & \frac{1}{2 i \pi} \int_{\gamma} c_{\sqrt{-z}}(t)(Q(t)-z I)^{-1}\left(Q(0) \varphi-f^{*}(0)\right) d z \\
& +\frac{1}{2 i \pi} \int_{\gamma} c_{\sqrt{-z}}(t) Q(t)(Q(t)-z I)^{-1}\left(Q(0)^{-1}-Q(t)^{-1}\right) Q(0) \varphi d z \\
= & \Delta_{7}(t)+\Delta_{8}(t),
\end{aligned}
$$

so, for $0 \leq \tau<t \leq \delta$,

$$
\begin{aligned}
\Delta_{7}(t)-\Delta_{7}(\tau)= & \frac{1}{2 i \pi} \int_{\gamma} c_{\sqrt{-z}}(t)(Q(t)-z I)^{-1}\left(Q(0) \varphi-f^{*}(0)\right) d z \\
& -\frac{1}{2 i \pi} \int_{\gamma} c_{\sqrt{-z}}(\tau)(Q(\tau)-z I)^{-1}\left(Q(0) \varphi-f^{*}(0)\right) d z \\
= & \frac{1}{2 i \pi} \int_{\gamma} c_{\sqrt{-z}}(t)\left((Q(t)-z I)^{-1}-(Q(\tau)-z I)^{-1}\right)\left(Q(0) \varphi-f^{*}(0)\right) d z \\
& +\frac{1}{2 i \pi} \int_{\gamma}\left(c_{\sqrt{-z}}(t)-c_{\sqrt{-z}}(\tau)\right)(Q(\tau)-z I)^{-1}\left(Q(0) \varphi-f^{*}(0)\right) d z \\
= & \frac{1}{2 i \pi} \int_{\gamma} c_{\sqrt{-z}}(t)\left((Q(t)-z I)^{-1}-(Q(\tau)-z I)^{-1}\right)\left(Q(0) \varphi-f^{*}(0)\right) d z \\
& +\frac{1}{2 i \pi} \int_{\gamma}\left(c_{\sqrt{-z}}(t)-c_{\sqrt{-z}}(\tau)\right) \\
& \left.\left.\times\left\{(Q(\tau)-z I)^{-1}-Q(0)-z I\right)^{-1}\right)\left(Q(0) \varphi-f^{*}(0)\right)\right\} d z \\
& +\frac{1}{2 i \pi} \int_{\gamma}\left(c_{\sqrt{-z}}(t)-c_{\sqrt{-z}}(\tau)\right)(Q(0)-z I)^{-1}\left(Q(0) \varphi-f^{*}(0)\right) d z \\
\Delta_{7}(t)-\Delta_{7}(\tau):= & (\mathrm{I})+(\mathrm{II})+(\mathrm{III}) .
\end{aligned}
$$

Step 3. It is sufficient to study the Hölderian property of the term (III). Using 
the fact that

$$
f^{*}(0)=f(0)-\left.\left(\frac{d^{2}}{d t^{2}} Q(t)^{-1}\right)\right|_{t=0} Q(0) \varphi+r_{\delta}\left(\varphi, \psi^{*}, f^{*}\right)
$$

we get

$$
\begin{aligned}
(\mathrm{III})= & \frac{1}{2 i \pi} \int_{\gamma}\left(c_{\sqrt{-z}}(t)-c_{\sqrt{-z}}(\tau)\right)(Q(0)-z I)^{-1} \\
& \times\left(Q(0) \varphi+\left.\left(\frac{\partial^{2}}{\partial t^{2}} Q(t)^{-1}\right)\right|_{t=0} Q(0) \varphi-f(0)\right) d z \\
& +\frac{1}{2 i \pi} \int_{\gamma}\left(c_{\sqrt{-z}}(t)-c_{\sqrt{-z}}(\tau)\right)(Q(0)-z I)^{-1} r_{\delta}\left(\varphi, \psi^{*}, f^{*}\right) d z \\
(\mathrm{III}):= & (\mathrm{III})_{1}+(\mathrm{III})_{2} .
\end{aligned}
$$

But $\left\|(\mathrm{III})_{2}\right\|=O\left(|t-\tau|^{2 \alpha}\right)$, since it has been proved that $r_{\delta}\left(\varphi, \psi^{*}, f^{*}\right) \in D_{q(0)}(\alpha,+\infty)$.

On the other hand $\left\|(\mathrm{III})_{1}\right\|=O\left(|t-\tau|^{2 \theta}\right.$ ) if and only if (see Lemma 1.2)

$$
f(0)-Q(0) \varphi-\left.\frac{d^{2}(Q(t))^{-1}}{d t^{2}}\right|_{t=0} \varphi \in D_{q(0)}(\theta,+\infty) .
$$

\section{A model example}

Let $\Omega$ be an open regular domain of $\mathbb{R}^{3}$ with a $C^{\infty}$ boundary $\Gamma$. Denote by $\Omega^{\delta}$ the thin layer

$$
\Omega^{\delta}=\{x=m+t \mathbf{n}(m): \quad m \in \Gamma, \quad t \in] 0, \delta[\},
$$

where $\delta>0$ is a parameter destined to tend to 0 and by $\mathbf{n}(m)$, the unit normal at the point $m$ of $\Gamma$ outwardly oriented.

Consider the boundary value problem

$$
(P) \begin{cases}\Delta v-\lambda v=0 & \text { in } \Omega^{\delta} \\ v=\varphi & \text { on } \Gamma^{0}=\Gamma \times\{0\}, \\ \frac{\partial v}{\partial \mathbf{n}}=0 & \text { on } \Gamma^{\delta}=\Gamma \times\{\delta\} .\end{cases}
$$

We associate to the function $v$ defined on $\Omega^{\delta}$ the function $u$ defined on $\left.\Gamma \times\right] 0,1[$ by $u(m, t):=v(x)$.

Let $\mathcal{R}(m)$ be the symmetric operator of the tangent plane $T_{m}(\Gamma)$ which characterizes the curvature of $\Gamma$ at the point $m$. For $\delta$ small enough, the operator $I+t \mathcal{R}(m)$ is an automorphism of the tangent plane $T_{m}(\Gamma)$. 
Then we can write

$$
\begin{aligned}
& \nabla v(x)=(I+t \mathcal{R}(m))^{-1} \nabla_{\Gamma} u(m, t)+\frac{\partial u}{\partial t}(m, t) \mathbf{n}(m), \\
& \Delta v(x)=\frac{\operatorname{div}_{\Gamma}\left[\operatorname{det}(I+t \mathcal{R}(m))(I+t \mathcal{R}(m))^{-2} \nabla_{\Gamma} u(m, t)\right]}{\operatorname{det}(I+t \mathcal{R}(m))},
\end{aligned}
$$

where $\nabla_{\Gamma}$ and $\operatorname{div}_{\Gamma}$ denote respectively the tangential surface gradient operator and the tangential surface divergence operator on $\Gamma$.

We have

$$
\operatorname{det}(I+t \mathcal{R}(m))=1+2 t H(m)+t^{2} G(m),
$$

with

$$
2 H(m)=\operatorname{tr} \mathcal{R}(m), \quad G(m)=\operatorname{det} \mathcal{R}(m) .
$$

Observe that, when $\delta$ is small enough, $\operatorname{det}(I+t \mathcal{R}(m)) \neq 0$ and there exists a constant $C>0$ such that

$$
\forall(m, t) \in \Gamma \times] 0, \delta\left[\quad|\operatorname{det}(I+t \mathcal{R}(m))|=\left|1+2 t H(m)+t^{2} G(m)\right| \geq C .\right.
$$

Put now $E=L^{p}(\Gamma)$ and denote by $u$ the vectorial function $\left.u:\right] 0, \delta[\rightarrow E$ defined by

$$
u(t)(m)=u(m, t) .
$$

Define the bounded multiplication operator $p(t)$ by

$$
\left\{\begin{array}{l}
D(p(t))=L^{p}(\Gamma) \\
(p(t) \psi)(m)=\frac{\frac{\partial}{\partial t} \operatorname{det}(I+t \mathcal{R}(m))}{\operatorname{det}(I+t \mathcal{R}(m))} \psi(m)=\frac{2 H(m)+2 t G(m)}{1+2 t H(m)+t^{2} G(m)} \psi(m),
\end{array}\right.
$$

and the unbounded (elliptic) operator $q(t)$ by

$$
\left\{\begin{array}{l}
D(q(t))=W^{2, p}(\Gamma) \\
(q(t) \psi)(m)=\frac{\operatorname{div}_{\Gamma}\left[\operatorname{det}(I+t \mathcal{R}(m))(I+t \mathcal{R}(m))^{-2} \nabla_{\Gamma} \psi(m)\right]}{\operatorname{det}(I+t \mathcal{R}(m))}-\lambda \psi(m) .
\end{array}\right.
$$

Then problem $(P)$ can be written in the following abstract differential boundary problem:

$$
\left\{\begin{array}{l}
\left.u^{\prime \prime}(t)+p(t) u^{\prime}(t)+q(t) u(t)-\lambda u(t)=0 \text { on }\right] 0, \delta[ \\
u(0)=\varphi \\
u^{\prime}(\delta)=0
\end{array}\right.
$$

All the preceding abstract results apply to this model example as we will prove it in the forthcoming part II.

Notice that this problem has also been considered by Favini et al. [5], in the framework of the interpolation space $\left.W^{\theta}\left(0, \delta ; L^{p}(\Gamma)\right), \theta \in\right] 0,1[, p \in] 1, \infty[$, in the case of constant operator coefficients. 


\section{References}

[1] P. Acquistapace, Some existence and regularity results for abstract nonautonomous parabolic equations, J. Math. Anal. Appl. 99 (1984), no. 1, 9-64.

[2] A. Bendali and K. Lemrabet, The effect of a thin coating on the scattering of a time-harmonic wave for the Helmholtz equation, SIAM J. Appl. Math. 56 (1996), no. 6, 1664-1693.

[3] A. Favini, R. Labbas, S. Maingot, H. Tanabe, and A. Yagi, On the solvability and the maximal regularity of complete abstract differential equations of elliptic type, Funkcial. Ekvac. 47 (2004), no. $3,423-452$.

[4] Complete abstract differential equations of elliptic type in UMD spaces, Funkcial. Ekvac. 49 (2006), no. 2, 193-214.

[5] A. Favini, R. Labbas, K. Lemrabet, and S. Maingot, Study of the limit of transmission problems in a thin layer by the sum theory of linear operators, Rev. Mat. Complut. 18 (2005), no. 1, 143-176.

[6] G. Da Prato and P. Grisvard, Sommes d'opérateurs linéaires et équations différentielles opérationnelles, J. Math. Pures Appl. (9) 54 (1975), no. 3, 305-387.

[7] P. Grisvard, Spazi di tracce e applicazioni, Rend. Mat. (6) 5 (1972), 657-729 (Italian, with English summary).

[8] M. Haase, The functional calculus for sectorial operators and similarity methods, Ph.D. Thesis, Universität Ulm, Germany, 2003.

[9] R. Labbas, Thèse d'état, Université de Nice, june 1987.

[10] A. Lunardi, Analytic semigroups and optimal regularity in parabolic problems, Progress in Nonlinear Differential Equations and Their Applications, vol. 16, Birkhäuser, Basel, 1995.

[11] E. Sinestrari, On the abstract Cauchy problem of parabolic type in spaces of continuous functions, J. Math. Anal. Appl. 107 (1985), no. 1, 16-66.

[12] H. Tanabe, Equations of evolution, translated by N. Mugibayashi and H. Haneda, Monographs and Studies in Mathematics, vol. 6, Pitman, Boston, Mass., 1979.

[13] A. Yagi, On the abstract evolution equation of parabolic type, Osaka J. Math. 14 (1977), no. 3, $557-568$. 